\title{
Phase Transitions and Percolation at Criticality in Enhanced Random Connection Models
}

\author{
Srikanth K. Iyer ${ }^{1}$. Sanjoy Kr. Jhawar ${ }^{1}$
}

Received: 11 September 2020 / Accepted: 23 September 2021 / Published online: 13 January 2022

(C) The Author(s), under exclusive licence to Springer Nature B.V. 2021

\begin{abstract}
We study phase transition and percolation at criticality for three random graph models on the plane, viz., the homogeneous and inhomogeneous enhanced random connection models (RCM) and the Poisson stick model. These models are built on a homogeneous Poisson point process $\mathcal{P}_{\lambda}$ in $\mathbb{R}^{2}$ of intensity $\lambda$. In the homogeneous RCM, the vertices at $x, y$ are connected with probability $g(|x-y|)$, independent of everything else, where $g:[0, \infty) \rightarrow[0,1]$ and $|\cdot|$ is the Euclidean norm. In the inhomogeneous version of the model, points of $\mathcal{P}_{\lambda}$ are endowed with weights that are non-negative independent random variables with distribution $P(W>w)=w^{-\beta} 1_{[1, \infty)}(w)$, $\beta>0$. Vertices located at $x, y$ with weights $W_{x}, W_{y}$ are connected with probability $1-\exp \left(-\frac{\eta W_{x} W_{y}}{|x-y|^{\alpha}}\right), \eta, \alpha>0$, independent of all else. The graphs are enhanced by considering the edges of the graph as straight line segments starting and ending at points of $\mathcal{P}_{\lambda}$. A path in the graph is a continuous curve that is a subset of the union of all these line segments. The Poisson stick model consists of line segments of independent random lengths and orientation with the mid point of each segment located at a distinct point of $\mathcal{P}_{\lambda}$. Intersecting lines form a path in the graph. A graph is said to percolate if there is an infinite connected component or path. We derive conditions for the existence of a phase transition and show that there is no percolation at criticality.
\end{abstract}

Keywords Random geometric graphs · Random connection model ·

Enhanced random connection model · Percolation · Phase transition ·

Continuity of the percolation function

Mathematics Subject Classification (2010) Primary: 82B43 - 60D05; Secondary: 05C80

SKI's research was supported in part by SERB Matrics grant MTR/2018/000496 and DST-CAS.

SKJ's research was supported by DST-INSPIRE Fellowship.

Srikanth K. Iyer

srikiyer@gmail.com

Extended author information available on the last page of the article. 


\section{Introduction and Main Results}

The study of random graphs started with the pioneering work by Erdős and Rényi $[15,16]$ and Gilbert [21] on the Erdős-Rényi model. The random graph in the ErdôsRényi model is constructed on a set of $n$ vertices, for some $n \in \mathbb{N}$ with an edge drawn between any two pairs of nodes independently with probability $p \in[0,1]$. Detailed work on the Erdős-Rényi graph can be found in [7, 30, 32]. The Bernoulli lattice percolation model on $\mathbb{Z}^{d}$ is an extensively studied random graph model [24, $33,34]$ where the geometry of the underlying space plays an important role. The vertex set is $\mathbb{Z}^{d}$ with an edge between points at Euclidean distance one with probability $p$ independent of other edges. The above geometric model was extended to the continuum by considering a point process in $\mathbb{R}^{d}$ with edges between points that are within a Euclidean distance $r>0$. Such a model has found wide application in modeling ad-hoc wireless networks and sensor networks. This model is called the Boolean model [22] or the random geometric graph (RGG). The questions of interest in such applications are percolation, connectivity and coverage, details of which can be found in [20] and [25]. Rigorous theoretical analysis of the percolation problem in such graphs can be found in [39] while the monograph [42] carries a detailed compilation of the important results on the topic in the sparse, thermodynamic and connectivity regimes. When each point of the underlying point process has an independent subset of $\mathbb{R}^{d}$ associated with it, then the union of all such sets is what forms the germ-grain model which is of much interest in stochastic geometry [48].

The main goal of this paper is to derive conditions under which three network models on the plane exhibit a phase transition and show that under some additional conditions the percolation function is continuous. We shall now describe these models and the problem of interest and discuss some applications. All these three models are constructed over a homogeneous Poisson point process denoted $\mathcal{P}_{\lambda}$ in $\mathbb{R}^{2}$ with intensity parameter $\lambda>0$. A phase transition refers to the abrupt emergence of an infinite component in the graph, in which case we say that the graph percolates. A phase transition is said to occur if there exists a critical value $\lambda_{c} \in(0, \infty)$ of $\lambda$ such that for $\lambda>\lambda_{c}$ the random graph under consideration percolates and for $\lambda<\lambda_{c}$ the random graph does not percolate. It can be shown using ergodicity that for $\lambda>\lambda_{c}$, there is an infinite component with probability one. In many of the percolation models in $\mathbb{R}^{d}$ it can also be shown that there is unique infinite component by adapting the Burton-Keane argument [24, 39]. The percolation function refers to the probability that a typical vertex in the graph is part of the infinite component. Percolation is equivalent to the percolation function being positive. The continuity of the percolation function is a problem of much interest in the random graph literature. See for instance, [14] for a new proof showing absence of percolation at criticality in the Bernoulli bond percolation model on $\mathbb{Z}^{d}$ with $d=2$. An entirely different technique known as the lace expansion can be used in higher dimensions. This method was first used by Hara and Slade in [26] and [27] to prove continuity of the percolation function for dimensions $d \geqslant 19$. Fitzner and Hofstad in [18] obtained a significant improvement by showing that the lace expansion works for $d \geqslant 11$. The problem still remains open for $3 \leqslant d \leqslant 10$. We refer the reader to the book by Heydenreich and Hofstad [28] for a concise collection of recent results on high-dimensional 
percolation. In a recent work Heydenreich et al. [29] describe the lace expansion method and mean-field behavior for the random connection model.

The random connection model (RCM) is a generalization of the RGG and a continuum version of the long range percolation on lattices. This model was introduced in [43] and later studied in the context of wireless networks. In such networks communication between nodes depends on the distance between the nodes as well as the interference coming from transmissions from other nodes in the network [38, 39]. In the random connection model we consider, the vertex set will be a homogeneous Poisson point process denoted $\mathcal{P}_{\lambda}$ in $\mathbb{R}^{d}$ with intensity parameter $\lambda$ for some $\lambda>0$. An undirected edge denoted $\{x, y\}$ exists between vertices located at $x, y$ with probability $g(|x-y|)$ independent of everything else, where $g:[0, \infty) \rightarrow$ $[0,1]$ is non-increasing. We denote this graph by $G_{\lambda}$. Penrose in [43] showed that a phase transition occurs in $G_{\lambda}$ if and only if the connection function satisfies $0<\int_{\mathbb{R}^{d}} g(|x|) d x<\infty$.

The first model that we consider is the enhanced RCM. To define this model consider the RCM described above on the plane $(d=2)$. If an edge $\{x, y\}$ exists in the RCM then we refer to $x$ and $y$ as direct neighbors. We view each edge as a straight line segment and denote it by $\overline{x y}$. For any two edges $\left\{x_{1}, x_{2}\right\}$ and $\left\{x_{3}, x_{4}\right\}$ in the RCM that intersect, we say that the vertices $x_{1}, x_{2}$ are indirect neighbors of $x_{3}, x_{4}$ and vice versa. We will refer to the resulting graph as the enhanced random connection model (eRCM) and denote it by $G_{\lambda}^{e}$. It will be more useful to think of the eRCM as enhancing the available paths in the network rather than introducing additional edges as can be seen from the following applications. Intersecting edges along a path in the RCM allow for switching from one path (in the original graph) to another. The eRCM can be considered as a model for a road or a pipeline network where connections are made locally and intersecting roads or pipelines allow the traffic or the fluid to switch paths. The above could also be used as a model for thin slab of porous media where connections between nodes resemble pipes and crossing of these pipes allow the fluid to flow from one pipe to another. An alternate model for road networks was studied in $[2,3]$. The construction of an optimal road network by using the trade-off between a measure of shortness of route and normalized network length for a one parameter family of proximity graphs is studied in [3]. In [2] the author introduces scale invariant spatial networks whose primitives are the routes between points on the plane. The problems of interest are the existence and uniqueness of infinite geodesics, continuity of routes as a function of end points and the number of routes between distant sets on the plane.

In this context it would be more appropriate to consider an inhomogeneous version of the eRCM model where each vertex is endowed with a weight that is indicative of the size, importance of a city or town. In the basic inhomogeneous model we consider, the vertex set is an independent marking of $\mathcal{P}_{\lambda}$ with weight distribution given by $P(W>w)=w^{-\beta} 1_{[1, \infty)}(w)$ for some $\beta>0$. Vertices located at $x, y \in \mathbb{R}^{2}$ endowed with random weights $W_{x}, W_{y}$ are connected by an edge independently with probability

$$
g(x, y)=1-\exp \left(-\frac{\eta W_{x} W_{y}}{|x-y|^{\alpha}}\right)
$$


where $\eta, \alpha$ are positive constants. The graph thus obtained is then enhanced in the same manner as described above to obtain the inhomogeneous eRCM (ieRCM). This is the second model that we shall study in this paper. We denote the random graph obtained in the inhomogeneous RCM and the enhanced inhomogeneous RCM by $H_{\lambda}, H_{\lambda}^{e}$ respectively. Percolation properties for inhomogeneous random connection model with this type of inhomogeneity was studied for long range percolation model on $\mathbb{Z}^{d}$ by Deprez et al. in [12] and in the continuum for fixed intensity $\lambda$ by Deprez and Wüthrich in [13]. Phase transition is expressed in terms of the parameter $\eta$ instead of $\lambda$. A simple scaling argument shows that these two are equivalent. In both these models, a phase transition is shown to occur for $d=1$ only if $\alpha \beta>2$ and $1<\alpha<2$ and for $d \geqslant 2$ only if $\alpha>d$ and $\alpha \beta>2 d$. For all $d$ the percolation function has been shown to be continuous under the condition that $\alpha \beta>2 d$ and $\alpha \in(d, 2 d)$. For $d \geqslant 2$ the case when $\min \{\alpha, \alpha \beta\}>2 d$ is open.

The third random graph model on the plane we shall analyze is the Poisson stick model. This is an example of a model that satisfies the axiomatic conditions of so called scale invariant spatial networks mentioned above. This model which was introduced in [46] consists of sticks of independent random lengths whose mid points are located at points of $\mathcal{P}_{\lambda}$ with each stick having a random independent orientation. The half-length of the sticks were assumed to have a density with bounded support. Two points in $\mathcal{P}_{\lambda}$ are neighbors in the resulting graph provided the corresponding sticks intersect. A phase transition was shown to occur in such a graph. The Poisson stick graph appears to be a natural model for a network structure formed by silicon nanowires and carbon and other nanotubes on the surface of substrates. Percolation, conductance and many other significant properties of these nanowire networks are studied in [6, 31, 40, 44, 49]. In this paper we consider the Poisson stick model with stick-length distribution having unbounded support and orientation distributed according to some arbitrary non-degenerate distribution. We study existence of phase transition and the continuity of the percolation function. The enhancement in our first two models has similarity with the Steiner tree. The Poisson stick model is similar to the Poisson line process on the plane. The Poisson line process and the Steiner tree have been used by Aldous and Kendall in [4] to establish asymptotics of excess route length in arbitrary graphs.

\subsection{Notations}

We gather much of the notations we need here for easy reference. We define the notations with reference to the RCM and eRCM. However, they carry over to the ieRCM and the Poisson stick models in the obvious way. Let $C(x)\left(C^{e}(x)\right)$ be the connected component containing $x \in \mathcal{P}_{\lambda}$ in $G_{\lambda}\left(G_{\lambda}^{e}\right)$. Without loss of generality we assume that there is a vertex at the origin $O$, that is, we consider the process $\mathcal{P}_{\lambda}$ under the Palm measure $P^{o}$, the probability distribution conditioned on a point being at origin. The distribution of $\mathcal{P}_{\lambda}$ under $P^{o}$ is the same as that of $\mathcal{P}_{\lambda} \cup\{O\}$ under the original measure $P$. Let $C:=C(O), C^{e}:=C^{e}(O)$ and define the percolation probabilities for $G_{\lambda}, G_{\lambda}^{e}$ as

$$
\theta(\lambda):=P^{o}(|C|=\infty) \text { and } \theta^{e}(\lambda):=P^{o}\left(\left|C^{e}\right|=\infty\right)
$$


The percolation thresholds denoted by $\lambda_{c}, \lambda_{c}^{e}$ for the graphs $G_{\lambda}$ and $G_{\lambda}^{e}$ respectively are defined as

$$
\lambda_{c}:=\inf \{\lambda>0: \theta(\lambda)>0\} \text { and } \lambda_{c}^{e}:=\inf \left\{\lambda>0: \theta^{e}(\lambda)>0\right\} .
$$

Similarly, let $\tilde{\lambda}_{c}, \tilde{\lambda}_{c}^{e}, \lambda_{P S}$ be the percolation thresholds for the random graphs $H_{\lambda}, H_{\lambda}^{e}$ and $P S_{\lambda}$ respectively.

As mentioned earlier, since we work with paths in the graph, we shall often view the enhanced models as providing additional paths in the original graphs rather than adding edges. In this view, edges in the graphs $G_{\lambda}, H_{\lambda}$ are straight line segments joining the vertices of $\mathcal{P}_{\lambda}$.

Definition 1.1 (Path) Given any of the graphs $G_{\lambda}^{e}, H_{\lambda}^{e}$ or $P S_{\lambda}$ and $x, y \in \mathbb{R}^{2}$ we say that there is a path from $x$ to $y$ if there exists a closed continuous curve from $x$ to $y$ contained entirely in $\cup_{i=1}^{n} e_{i}$ for some edges $e_{1}, e_{2} \ldots, e_{n}$ in the case of $G_{\lambda}, H_{\lambda}$ and sticks in the case of $P S_{\lambda}$. Paths thus need not start or end at vertices in the graph.

Definition 1.2 (Crossing events) A path is said to cross a box $[a, b] \times[c, d]$ if the path is completely contained within the box with end points on opposite sides. We shall refer to these paths as crossings (see Fig. 1). For $s>0$ and $\rho>1$ let $L R_{S}(\rho)$ be the event that there exists a crossing along the longer side (left-to-right) of the rectangle $[0, \rho s] \times[0, s]$ and $T D_{s}(\rho)$ be the event that there exists a crossing along the shorter side (top-to-down) of the rectangle $[0, \rho s] \times[0, s] . C_{s}(\rho):=P\left(L R_{s}(\rho)\right)$.

\subsection{Main Results}

Our first result is on the existence of a phase transition in the three models described earlier. Penrose [43] showed that there is a non-trivial phase transition in the RCM, that is, $0<\lambda_{c}<\infty$ under the condition $0<\int_{0}^{\infty} r g(r) d r<\infty$. We now prove a similar result for the eRCM albeit under a stronger restriction on $g$. In case of the ieRCM the condition required is stronger than the one for the iRCM as derived in [13]. Roy [46] showed the existence of a phase transition in the Poisson stick model

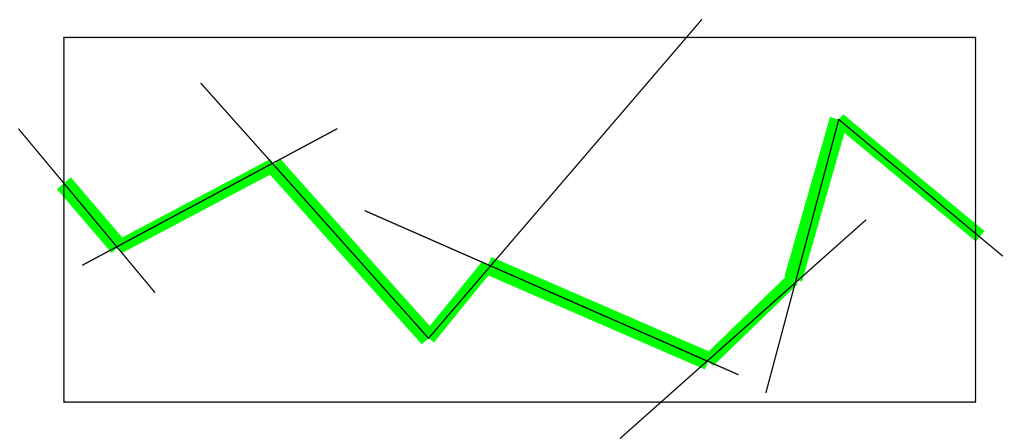

Fig. 1 The green curve is a left-right crossing of the box 
under the assumption that the stick length distribution has bounded support, a result which we extend to sticks of unbounded lengths.

\section{Theorem 1.1 A phase transition occurs in the}

(i) eRCM $G_{\lambda}^{e}$ if the connection function $g$ satisfies $0<\int_{0}^{\infty} r^{3} g(r) d r<\infty$.

(ii) ieRCM $H_{\lambda}^{e}$ with the connection function of the form (1.1) if $\alpha>4$ and $\alpha \beta>8$. (iii) graph $P S_{\lambda}$ with half-length density $h$ if $0<\int_{0}^{\infty} \ell^{2} h(\ell) d \ell<\infty$.

The condition for the existence of an infinite component for large intensities is obtained by comparing the enhanced model with the usual non-enhanced version. For the other side we shall show that the probability that there exists a self avoiding path of length $n$ converges to zero as $n \rightarrow \infty$.

Our next result establishes a RSW lemma which is one of the most useful result in planar percolation models. It states that if the probability of crossing a square is uniformly bounded away from zero then so is the probability of crossing a rectangle along the longer side. We demonstrate its utility by establishing that percolation does not occur at criticality. The RSW lemma was first proved for the independent Bernoulli bond percolation model on the $\mathbb{Z}^{2}$ lattice independently by Russo [47] and Seymour and Welsh [50]. A similar result about occupied and vacant crossings was proved for the Boolean model on $\mathbb{R}^{2}$ by Roy [45]. In [46] an RSW result has been proved for the Poisson stick model with sticks of bounded lengths. The RSW results in this article are analogous to those in [51] for the percolation model on Poisson-Voronoi tessellations in $\mathbb{R}^{2}$. RSW results, continuity of critical parameter and sharpness of the phase transition for the Boolean model with unbounded radius distribution has been studied by Ahlberg et al. in [1]. For the continuum percolation model with random ellipses on the plane, percolation and connectivity behavior of the vacant and covered set has been studied by Teixeira et al. in [52].

We prove the RSW lemma under the condition that the connection function in the eRCM is of the form $g(r)=O\left(r^{-c}\right)$ as $r \rightarrow \infty$ and the half-length density $h$ satisfies $h(\ell)=O\left(\ell^{-c}\right)$ as $\ell \rightarrow \infty$. This assumption is required in order to derive an estimate on the longest edge/stick length intersecting a large box. By Theorem $1.1 \mathrm{a}$ phase transition occurs under the above assumptions in the eRCM provided $c>4$ and in $P S_{\lambda}$ if $c>3$. The first two assertions in Theorem 1.2 are equivalent formulations of the RSW lemma while the third is derived as a (non-trivial) corollary of the first assertion. The RSW results are derived by adapting the technique developed by Tassion in [51] for Poisson-Voronoi percolation. The presence or absence of events in disjoint regions of space that are determined by the color of the cells in those regions are independent provided there are sufficiently many points of the point process in the space surrounding these cells. This argument does not carry over to the case of the random graph models we study here, since the edges are of unbounded lengths. We first need a control on the length of the long edges. Even so, the absence of long edges in two disjoint regions far apart is not determined by the point process over any suitable disjoint regions. Thus a different argument is required to make the proof work. We then use a renormalization technique similar to the one used in [5] (see also [10]) to show that the parameter set over which percolation occurs is open. We shall 
prove the results in detail for the eRCM. Much of the proof carries over to the other two models for which we will provide only the necessary details.

Theorem 1.2 Suppose the following conditions hold.

(I) In the eRCM $G_{\lambda}^{e}$ the connection function $g$ satisfies $g(r)=O\left(r^{-c}\right)$ as $r \rightarrow \infty$ with $c>4$.

(II) In the ieRCM $H_{\lambda}^{e}$ the connection function $g$ is of the form (1.1) with $\min \{\alpha, \alpha \beta\}>4$.

(III) In the graph $P S_{\lambda}$ with half-length density $h$ satisfies $h(\ell)=O\left(\ell^{-c}\right)$ with $c>3$.

Then the following conclusions hold for all the three graphs $G_{\lambda}^{e}, H_{\lambda}^{e}$ and $P S_{\lambda}$.

(i) If $\inf _{s \geqslant 1} C_{s}(1)>0$ then for any $\rho \geqslant 1, \inf _{s \geqslant 1} C_{s}(\rho)>0$.

(ii) If $\lim _{s \rightarrow \infty} C_{s}(1)=1$ then for any $\rho \geqslant 1, \lim _{s \rightarrow \infty} C_{s}(\rho)=1$.

(iii) The percolation function is continuous.

Remark 1.1 The inhomogeneous RCM has also been studied in [8, 23] and [35] with a slightly different but equivalent connection function. In this alternate set-up two vertices located at $x, y$ are connected with probability

$$
g(x, y)=1-\exp \left(-\left(\frac{\eta W_{x} W_{y}}{|x-y|^{2}}\right)^{\alpha}\right),
$$

where the distribution of weights is as defined above (1.1). Setting $V_{x}=W_{x}^{\alpha}$ and $\alpha^{\prime}=2 \alpha$ the model prescribed by (1.4) reduces to the model specified by (1.1) with parameters $\alpha^{\prime}$ and $\beta / \alpha$. It is then easy to derive the equivalent conditions on the parameters under the connection function given by (1.4). The condition required for Theorem 1.1 ( $i$ i) to hold will be $\alpha>2, \beta>4$ and for Theorem 1.2 (II) to hold becomes $\alpha>2$ and $\beta>2$. This is the regime in which the weights have finite variance and the random graph has fewer long-range edges.

Remark 1.2 To prove part (iii) of Theorem 1.2 it suffices to show that there is no percolation at criticality, that is, $\theta^{e}\left(\lambda_{c}^{e}\right)=0$. Indeed, for $\lambda<\lambda_{c}^{e}, \theta^{e}(\lambda) \equiv 0$. Continuity of the percolation function in the supercritical regime $\left(\lambda>\lambda_{c}^{e}\right)$ follows along the same lines as in the proof of Theorem 8.8 in [24]. Right continuity of $\theta^{e}(\lambda)$ is a simple consequence of monotonicity (see Lemma 8.9 in [24]). Left continuity can be proved by adapting Lemma 8.10 in [24]. The proof relies on the uniqueness of infinite component. Uniqueness can be proved using the same arguments as in Theorem 6.3 in [39].

\subsection{Open Problems}

A natural first question is to examine whether $\lambda_{c}^{e}<\lambda_{c}$ and $\tilde{\lambda}_{c}^{e}<\tilde{\lambda}_{c}$. In a recent work [17], sharpness of phase transition has been proved for the RCM under the condition that edge lengths are bounded. Such results are proved on the plane for the Poisson Boolean model with unbounded grain size under certain moment conditions and for 
the Poisson-Voronoi percolation using the RSW Lemma [1]. Under what conditions would such a result hold for the eRCM?

\section{The Enhanced Random Connection Model}

In this section we shall prove the non-trivial phase transition (Theorem 1.1 $(i)$ ), the RSW result and non-percolation at criticality (Theorem 1.2) for the eRCM. Much of the proof carries over to the other two models, i.e., the inhomogeneous eRCM and the Poisson stick model, for which we will provide only the necessary details.

In what follows $c_{0}, c_{1}, c_{2}, \ldots$ and $C_{1}, C_{2}, \ldots$ will denote constants whose values will change from place to place. $|\cdot|$ will be used to refer to the Euclidean norm, the cardinality of a set as well as the Lebesgue measure.

\subsection{Proof of Theorem 1.1 (i)(Non-trivial phase transition)}

It is clear from the definition that $G_{\lambda}^{e}$ percolates if $G_{\lambda}$ does. So we have $\lambda_{c}^{e} \leqslant \lambda_{c}$. From Theorem 1 in Penrose [43] we know that $\lambda_{c} \in(0, \infty)$ iff $\int_{0}^{\infty} r g(r) d r \in$ $(0, \infty)$. Since $g(r) \in[0,1], \int_{0}^{\infty} r^{3} g(r) d r \in(0, \infty)$ implies $\int_{0}^{\infty} r g(r) d r \in(0, \infty)$. It follows from the above observations that $\lambda_{c}^{e}<\infty$.

We now show that $\lambda_{c}^{e}>0$. We shall bound the probability that there is a selfavoiding path formed using $n$ distinct points of $\mathcal{P}_{\lambda}$ starting from the origin. For any $\mathbf{x}=\left(x_{1}, x_{2}, \ldots, x_{n}\right)$ of $n$ distinct points of the Poisson point process, we examine whether there is a path starting at $x_{0}=O$ and uses edges (or parts of it) with end points from $\mathbf{x}$ in the coordinate order. We shall denote any such path by $x_{0} \rightarrow x_{1} \rightarrow$ $x_{2} \rightarrow \ldots \rightarrow x_{n}$ even though some of these points may not be part of the path. Since we need only an upper bound on the percolation probability, we allow our self-avoiding paths to have loops. However, each edge or a part of it is used exactly once while traversing the path. For each ordered sequence $\mathbf{x}$ as above, there can be several ways in which a path can occur. See Fig. 2 for self-avoiding paths formed by $\left(x_{1}, x_{2}, x_{3}, x_{4}\right)$. Each such possibility gives rise to a unique block structure that we describe below. In order to carry out the computation we segregate all paths in disjoint block structures.

We now illustrate this via an example. Take $n=4, x_{0}=O$ and $x_{1}, x_{2}, x_{3}, x_{4}$ be four distinct points in $\mathcal{P}_{\lambda}$. Suppose that $O \rightarrow x_{1} \rightarrow x_{2} \rightarrow x_{3} \rightarrow x_{4}$ is a self avoiding path. This can occur in only one way in the RCM (Fig. 2 (a)) but in the eRCM this can occur in three different ways (see Fig. 2). Note that in Fig. 2 (c) we allow the segment $\overline{x_{3} x_{4}}$ to intersect the segment $\overline{x_{0} x_{1}}$.

Let $E\left(G_{\lambda}\right)$ denote the edge set of the graph $G_{\lambda}$. Fix $n \in \mathbb{N}$ and let $\mathbf{x}=$ $\left(x_{1}, x_{2}, \ldots, x_{n}\right) \in \mathcal{P}_{\lambda, \neq}^{n}$ be an ordered collection of $n$ distinct points in $\mathcal{P}_{\lambda}$. Define the sub-collection of indices

$$
\begin{aligned}
& I(\mathbf{x}):=\left\{i \in[n-1]:\left\{x_{i-1}, x_{i}\right\},\left\{x_{i}, x_{i+1}\right\} \in E\left(G_{\lambda}\right)\right\}, \\
& J(\mathbf{x}):=\left\{i, i+1: i \in[n-2],\left\{x_{i-1}, x_{i}\right\},\left\{x_{i+1}, x_{i+2}\right\} \in E\left(G_{\lambda}\right), \overline{x_{i-1} x_{i}} \cap \overline{x_{i+1} x_{i+2}}=z_{i} \notin \mathcal{P}_{\lambda}\right\} .
\end{aligned}
$$




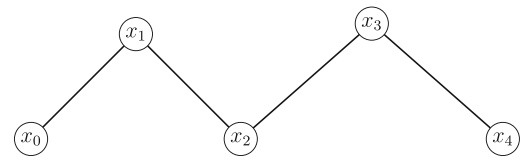

(a)

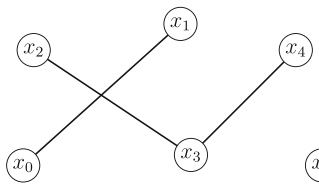

(b)

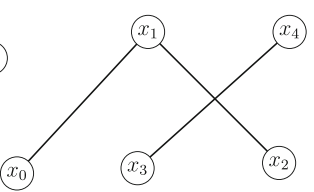

(c)

Fig. 2 All possible configurations of paths $x_{0} \rightarrow x_{1} \rightarrow x_{2} \rightarrow x_{3} \rightarrow x_{4}$

The last condition in the definition of $J(\mathbf{x})$ requires that the edges intersect at a point interior to both the edges. Suppose $I(\mathbf{x})=\left\{i_{1}, i_{2}, \ldots, i_{k}\right\}$ for some $0 \leqslant$ $k \leqslant n-1$, labeled in the increasing order. Set $i_{0}:=0, i_{k+1}:=n$ and define the blocks

$$
B_{j}(\mathbf{x}):=\left\{i_{j-1}<i<i_{j}: i \in J(\mathbf{x})\right\} \cup\left\{i_{j-1}, i_{j}\right\}, \quad 1 \leqslant j \leqslant k+1 .
$$

If $x_{0} \rightarrow x_{1} \rightarrow x_{2} \rightarrow \ldots \rightarrow x_{n}$ is a self avoiding path then $\cup_{j=0}^{k+1} B_{j}(\mathbf{x})=$ $\{0,1,2, \ldots, n\}$. If $i \in I(\mathbf{x})$ then $x_{i}$ lies on the path whereas if $i \in J(\mathbf{x})$ then the path uses a part of an edge one of whose end points is $x_{i}$. Let $B(\mathbf{x})=$ $\left(B_{1}(\mathbf{x}), \ldots, B_{k+1}(\mathbf{x})\right)$. With reference to Fig. 2, the diagram in (a) consists of four blocks $B_{i}(\mathbf{x})=\{i-1, i\}, 1 \leqslant i \leqslant 4$. The diagram in (b) has two blocks $B_{1}(\mathbf{x})=$ $\{0,1,2,3\}, B_{2}(\mathbf{x})=\{3,4\}$ while the one in (c) also has two blocks $B_{1}(\mathbf{x})=\{0,1\}$, $B_{2}(\mathbf{x})=\{1,2,3,4\}$.

Note that all blocks have even cardinality. For $0 \leqslant k \leqslant n-1, \mathcal{B}_{k}$ be the collection of all block structures $B=\left(B_{1}, \ldots, B_{k+1}\right)$ such that $\left|B_{j}\right|$ is even and for some $0<i_{1}<i_{2}<\cdots<i_{k}<n, B_{j}:=\left\{i: i_{j-1} \leqslant i \leqslant i_{j}\right\}$ with $i_{0}:=0, i_{k+1}:=n$. Let $B_{j}^{e}=\left\{i_{j-1}+2 r-1: r=1,2, \ldots,\left(i_{j}-i_{j-1}+1\right) / 2\right\}$ be the set of indices with an even ordering in $B_{j}$.

By definition of percolation probability the event that the origin lies in an infinite connected component implies that for each $n \in \mathbb{N}$ there is a self-avoiding path of length $n$ starting from the origin in $G_{\lambda}^{e}$.

$$
\begin{aligned}
\theta^{e}(\lambda) & \leqslant P^{o}\left(\text { there is a self-avoiding path on } n \text { vertices in } G_{\lambda}^{e} \text { starting at } x_{0}\right) \\
& \leqslant E^{o}\left[\sum_{\mathbf{x} \in \mathcal{P}_{\lambda, \neq}^{n}} 1\left\{x_{0} \rightarrow x_{1} \rightarrow x_{2} \rightarrow \cdots \rightarrow x_{n} \text { occurs }\right\}\right] \\
& =\sum_{k=0}^{n-1} \sum_{B \in \mathcal{B}_{k}} E^{o}\left[\sum_{\mathbf{x} \in \mathcal{P}_{\lambda, \neq}^{n}} 1\{B(\mathbf{x})=B\}\right]
\end{aligned}
$$

Recall that each $B \in \mathcal{B}_{k}$ is of the form $\left(B_{1}, \ldots, B_{k+1}\right)$. For a block of the form $B_{j}:=\left\{i: i_{j-1} \leqslant i \leqslant i_{j}\right\}$ of size larger than two, let

$A_{j}=\left\{\mathbf{y}=\left(y_{1}, \ldots, y_{n}\right): \overline{y_{i_{j-1}+2 r-2} y_{i_{j-1}+2 r-1}}\right.$ intersects $\overline{y_{i_{j-1}+2 r} y_{i_{j-1}+2 r+1}}$ for all $\left.r=1, \ldots,\left(i_{j}-i_{j-1}-1\right) / 2\right\}$ 
The intersection above is understood to be at an interior point of the line segments. For a block of size two we set $1_{A_{j}} \equiv 1$. Conditioning on $\mathcal{P}_{\lambda}$ and then applying the Campbell-Mecke formula we obtain

$$
\begin{aligned}
E^{o}\left[\sum_{\mathbf{x} \in \mathcal{P}_{\lambda, \neq}^{n}} 1\{B(\mathbf{x})=B\}\right] & =E^{o}\left[\sum_{\mathbf{x} \in \mathcal{P}_{\lambda, \neq}^{n}} E^{o}\left[\prod_{j=1}^{k+1} 1\left\{B_{j}(\mathbf{x})=B_{j}\right\} \mid \mathcal{P}_{\lambda}\right]\right] \\
& =E^{o}\left[\sum_{\mathbf{x} \in \mathcal{P}_{\lambda, \neq}^{n}} \prod_{j=1}^{k+1} \prod_{l \in B_{j}^{e}} g\left(x_{l-1}, x_{l}\right) 1_{A_{j}}(\mathbf{x})\right] \\
& =\lambda^{n} \underbrace{\iint \ldots \int}_{n \text { times }} \prod_{j=1}^{k+1} \prod_{l \in B_{j}^{e}} g\left(x_{l-1}, x_{l}\right) 1_{A_{j}}(\mathbf{x}) \prod_{l=1}^{n} d x_{l} .
\end{aligned}
$$

We now evaluate the contribution to (2.2) from blocks of various sizes. By contribution from a block we refer to the outcome of evaluating the integrals in (2.2) with respect to all variables with index in that block except for the one corresponding to the first index. We shall integrate the variables in the descending order starting with those in the block $B_{k+1}$. Blocks of size four and higher yield a nice formula for the upper bound. To see this one needs to compute the bound for a block of size six. We start with the simplest block of size two. The contribution from a block of size two will be of the form

$$
\int_{\mathbb{R}^{2}} g\left(\left|x_{1}-x_{2}\right|\right) d x_{2}=\int_{\mathbb{R}^{2}} g(|x|) d x=2 \pi \int_{0}^{\infty} r g(r) d r .
$$

Next we compute the contribution from a block of size four. For $a=\left(a_{1}, a_{2}\right), b=$ $\left(b_{1}, b_{2}\right)$, let $H^{+}(a, b):=\left\{\left(c_{1}, c_{2}\right) \in \mathbb{R}^{2}: \frac{c_{2}-a_{2}}{c_{1}-a_{1}} \geqslant \frac{a_{2}-b_{2}}{a_{1}-b_{1}}\right\}$. For $c \in H^{+}(a, b)$, let $D(a, b, c):=\left\{d \in \mathbb{R}^{2}: \overline{c d}\right.$ intersects $\left.\overline{a b}\right\}$. The contribution to (2.2) from a block of size four equals

$$
\int_{\mathbb{R}^{2}} g\left(\left|y_{1}-y_{2}\right|\right)\left(\int_{H^{+}\left(y_{1}, y_{2}\right)} \int_{D\left(y_{1}, y_{2}, y_{3}\right)} g\left(\left|y_{4}-y_{3}\right|\right) d y_{4} d y_{3}\right) d y_{2} .
$$

The region $D\left(y_{1}, y_{2}, y_{3}\right)$ is the region enclosed by the rays $\overrightarrow{y_{1} A}, \overrightarrow{y_{2} A^{\prime}}$ and the line segment $\overline{y_{1} y_{2}}$ (see Fig. 3). Suppose $\left|y_{1}-y_{2}\right|=\ell$. By a translation and rotation (so that $y_{1}$ is translated to the origin and $y_{2}$ to the point $\left.(\ell, 0)\right)$, we can write

$$
\int_{H^{+}\left(y_{1}, y_{2}\right)} \int_{D\left(y_{1}, y_{2}, y_{3}\right)} g\left(\left|y_{4}-y_{3}\right|\right) d y_{4} d y_{3}=\int_{\mathbb{R} \times\left\{a_{2}>0\right\}} \int_{D(O,(\ell, 0), a)} g(|a-b|) d b d a,
$$

where $a=\left(a_{1}, a_{2}\right)$ and $b=\left(b_{1}, b_{2}\right)$. Changing the variables $a, b$ to $u, v, r, \theta$ according to $a=(u+v \cos \theta, v \sin \theta), b=(u-(r-v) \cos \theta,-(r-v) \sin \theta)$ and noting that the determinant of the Jacobian satisfies $\left|J^{-1}\right|=r \sin \theta$ we can rewrite the right-hand side of (2.5) as

$$
\int_{0}^{\ell} \int_{0}^{\pi} \int_{0}^{\infty} \int_{0}^{r} g(r) r \sin \theta d v d r d \theta d u=2 \ell \int_{0}^{\infty} r^{2} g(r) d r .
$$




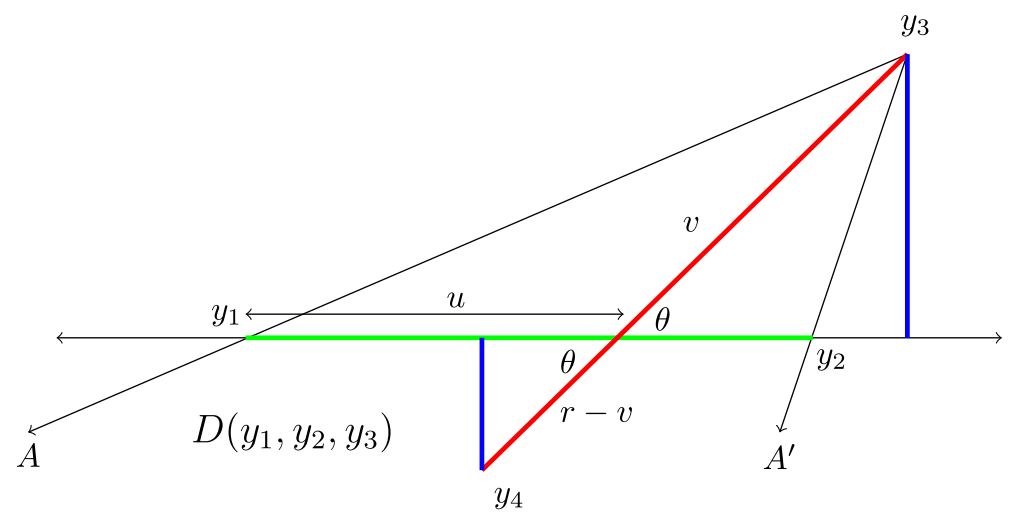

Fig. $3 D\left(y_{1}, y_{2}, y_{3}\right)$ is the unbounded region enclosed by the rays $\overrightarrow{y_{1} A}, \overrightarrow{y_{2} A^{\prime}}$ and the line segment $\overline{y_{1} y_{2}}$

By changing to polar coordinates and using (2.6) the expression in (2.4) equals

$$
\int_{0}^{\infty} \int_{0}^{2 \pi} \ell g(\ell)\left(2 \ell \int_{0}^{\infty} r^{2} g(r) d r\right) d \theta d \ell=4 \pi\left(\int_{0}^{\infty} r^{2} g(r) d r\right)^{2} .
$$

We shall write $H_{i, j}$ for $H^{+}\left(y_{i}, y_{j}\right)$ and $D_{i, j, k}$ for $D\left(y_{i}, y_{j}, y_{k}\right)$ for notational simplicity. The contribution from a block of size six can be found by applying the above procedure twice. Using (2.5), (2.6) we get

$$
\begin{aligned}
& \int_{\mathbb{R}^{2}} g\left(\left|y_{1}-y_{2}\right|\right)\left(\int_{H_{1,2}} \int_{D_{1,2,3}} g\left(\left|y_{4}-y_{3}\right|\right)\left(\int_{H_{3,4}} \int_{D_{3,4,5}} g\left(\left|y_{6}-y_{5}\right|\right) d y_{6} d y_{5}\right) d y_{4} d y_{3}\right) d y_{2} \\
& \leqslant \int_{\mathbb{R}^{2}} g\left(\left|y_{1}-y_{2}\right|\right)\left(\int_{H_{1,2}} \int_{D_{1,2,3}} g\left(\left|y_{4}-y_{3}\right|\right) 2\left|y_{4}-y_{3}\right| d y_{4} d y_{3}\right) d y_{2}\left(\int_{0}^{\infty} r^{2} g(r) d r\right) \\
& =2 \int_{\mathbb{R}^{2}} g\left(\left|y_{1}-y_{2}\right|\right) 2\left|y_{2}-y_{1}\right| d y_{2}\left(\int_{0}^{\infty} r^{3} g(r) d r\right)\left(\int_{0}^{\infty} r^{2} g(r) d r\right) \\
& =8 \pi\left(\int_{0}^{\infty} r^{2} g(r) d r\right)\left(\int_{0}^{\infty} r^{3} g(r) d r\right)\left(\int_{0}^{\infty} r^{2} g(r) d r\right) .
\end{aligned}
$$

By iterating the above procedure the contribution from the block of size $2 m+2$ on the vertices $\left\{y_{1}, y_{2}, \ldots, y_{2 m+2}\right\}$ where the edge $\overline{y_{i}, y_{i+1}}$ intersects $\overline{y_{i+2}, y_{i+3}}$ for all $i=1,2, \ldots, 2 m-1$ can be shown to be

$$
2^{m+1} \pi\left(\int_{0}^{\infty} r^{2} g(r) d r\right)\left(\int_{0}^{\infty} r^{3} g(r) d r\right)^{m-1}\left(\int_{0}^{\infty} r^{2} g(r) d r\right) .
$$

For a path with blocks $B_{1}, B_{2}, \ldots, B_{k+1}$ let

$$
k_{p}:=\left|\left\{j:\left|B_{j}\right|=2 p\right\}\right| \text { and } \bar{k}=\sum_{\substack{1 \leqslant j \leqslant n \\\left|B_{j}\right| \geqslant 6}}\left(\frac{\left|B_{j}\right|}{2}-2\right) .
$$


Since each vertex can be in at most two adjacent blocks we have that

$$
\bar{k} \leqslant \sum_{j=1}^{k+1} \frac{\left|B_{j}\right|}{2} \leqslant n .
$$

Substituting the contributions from each block $B_{1}, B_{2}, \ldots, B_{k+1}$, that is, the expression in (2.3), (2.7)-(2.9) in the expression on the right in (2.2) yields

$$
E^{o}\left[\sum_{\mathbf{x} \in \mathcal{P}_{\lambda, \neq}^{n}} 1\{B(\mathbf{x})=B\}\right]=\lambda^{n} \prod_{m=0}^{\left\lfloor\frac{n}{2}\right\rfloor}\left(2^{m+1} \pi\right)^{k_{m}}\left(\int_{0}^{\infty} r g(r) d r\right)^{k_{1}}\left(\int_{0}^{\infty} r^{2} g(r) d r\right)^{2\left(k-k_{1}\right)}\left(\int_{0}^{\infty} r^{3} g(r) d r\right)^{\bar{k}} .
$$

Using (2.11) in (2.12) and then using (2.2), (2.12) in (2.1) we obtain

$$
\theta^{e}(\lambda) \leqslant \lambda^{n} \sum_{k=0}^{n-1} \sum_{B \in \mathcal{B}_{k}}\left(\prod_{m=0}^{\left\lfloor\frac{n}{2}\right\rfloor}\left(2^{m+1} \pi\right)^{k_{m}}\right) C_{1}^{4 n}
$$

where $C_{1}=\max \left\{\int_{0}^{\infty} r^{j} g(r) d r, j=1,2,3\right\}$. Since $\sum_{m=1}^{\left\lfloor\frac{n}{2}\right\rfloor}(m+1) k_{m} \leqslant \sum_{m=1}^{\left\lfloor\frac{n}{2}\right\rfloor} 2 m k_{m} \leqslant$ $2 n$ and $\left|\mathcal{B}_{k}\right|=\left(\begin{array}{c}n-1 \\ k\end{array}\right)$, there exists a constant $C$ such that

$$
\theta^{e}(\lambda) \leqslant(C \lambda)^{n} \rightarrow 0
$$

as $n \rightarrow \infty$ for all $\lambda \in\left(0, \lambda_{0}\right)$, for some $\lambda_{0}>0$ provided $\max \left\{\int_{0}^{\infty} r^{j} g(r)\right.$ $d r, j=1,2,3\}<\infty$.

\subsection{Proof of Theorem 1.2 (i) (RSW)}

Before proceeding with the proof we recall some tools from Stochastic geometry, namely the Fortuin-Kasteleyn-Ginibre (FKG) inequality and the square-root trick that will be used in several places.

Increasing and Decreasing Events An event $A$ is said to be an increasing event if it continues to occur under addition of edges. An event $A$ is said to be a decreasing event if $A^{c}$ is increasing.

Theorem 2.1 (FKG inequality) If $A_{1}, A_{2}$ are both increasing or both decreasing events, then

$$
P\left(A_{1} \cap A_{2}\right) \geqslant P\left(A_{1}\right) P\left(A_{2}\right)
$$

Corollary 2.2 (Square-root trick) Let $A_{1}, A_{2}, \ldots, A_{k}$ be increasing events having equal probability. Then

$$
P\left(A_{1}\right) \geqslant 1-\left(1-P\left(\cup_{i=1}^{k} A_{i}\right)\right)^{\frac{1}{k}} .
$$


We shall provide a more general statement of the FKG inequality and its proof in Section 5 (Appendix). Some standard reference to the FKG inequality is [19] for discrete case and Theorem 2.2 in [39] for the continuum case. To prove the FKG inequality for increasing functionals on random geometric graphs we will need to combine the discrete FKG inequality with that for increasing functionals on Poisson point processes (Theorem 20.4, Last and Penrose [36]). For the square-root trick see Lemma 2.3 in [9].

Consider the graph $G_{\lambda}^{e}$ with connection function $g(r)=O\left(r^{-c}\right)$ as $r \rightarrow \infty$ where $c>4$ is arbitrary. Later we shall borrow some key results from Tassion [51] and hence will adopt many of the notations from that paper. A key ingredient in the proof is the following result on the length of the longest edge in $G_{\lambda}$ which allows us to localize the analysis. Let $B_{s}:=[-s, s]^{2}$ and for $t>s$ let $A_{s, t}:=B_{t} \backslash B_{s}$.

Proposition 2.3 For any $s>0$ let $M_{s}$ be the length of the longest edge in $G_{\lambda}$ intersecting the box $B_{s}=[-s, s]^{2}$. Suppose that the connection function $g$ satisfies $g(r)=O\left(r^{-c}\right)$ as $r \rightarrow \infty$. Then for any $c>4, t>0$ and $\tau>\frac{2}{c-2}$ we have $P\left(M_{t s}>s^{\tau}\right) \rightarrow 0$ as $s \rightarrow \infty$.

Proof of Proposition 2.3 Fix $c>4, t>0$. Let $B(O, s):=\left\{x \in \mathbb{R}^{2}:|x| \leqslant s\right\}$ be the ball of radius $s$ centered at the origin. Recall that for any two points $x, y \in \mathbb{R}^{2}, \overline{x y}$ denotes the line segment joining $x$ and $y$. Define the events $D_{s}(l)=\left\{M_{s}>l\right\}$, $O_{s}(\tau)=\left\{X \in \mathcal{P}_{\lambda}:\right.$ there is an edge of length longer than $s^{\tau}$ incident on $X$ in $\left.G_{\lambda}\right\}$ and

$\bar{O}_{t, s}(\tau)=\left\{(X, Y) \in \mathcal{P}_{\lambda}^{2}:\right.$ there is an edge in $G_{\lambda}$ joining $X, Y,|\overline{X Y}| \geqslant s^{\tau}, \overline{X Y}$ intersects $\left.B(O, \sqrt{2} s)\right\}$.

Then,

$$
\begin{aligned}
P\left(D_{t s}\left(s^{\tau}\right)\right) & \leqslant E\left[\sum_{X, Y \in \mathcal{P}_{\lambda}} 1\left\{\overline{X Y} \text { intersects } B_{t s}\right\} 1\left\{|X-Y| \geqslant s^{\tau}\right\}\right] \\
& \leqslant E\left[\sum_{X \in \mathcal{P}_{\lambda} \cap B(O, \sqrt{2} t s)} 1\left\{X \in O_{s}(\tau)\right\}\right]+E\left[\sum_{X, Y \in \mathcal{P}_{\lambda} \cap B(O, \sqrt{2} t s)^{c}} 1\left\{(X, Y) \in \bar{O}_{t, s}\right\}\right] .
\end{aligned}
$$

The Campbell-Mecke formula applied to the first term on the right-hand side of the last inequality in (2.16) yields

$$
\begin{aligned}
& E\left[\sum_{X \in \mathcal{P}_{\lambda} \cap B(O, \sqrt{2} t s)} 1\left\{X \in O_{s}(\tau)\right\}\right] \\
& =C \lambda(t s)^{2} P^{o}\left(O \in O_{s}(\tau)\right) \\
& \left.=C \lambda(t s)^{2}\left(1-P^{o} \text { (none of the edges incident on } \mathrm{O} \text { is of length } \geqslant s^{\tau}\right)\right) \\
& =C \lambda(t s)^{2}\left(1-\exp \left(-\lambda \int_{B\left(O, s^{\tau}\right)^{c}} g(|x|) d x\right)\right) \\
& \leqslant C(\lambda t s)^{2} \int_{B\left(O, s^{\tau}\right)^{c}} g(|x|) d x=C_{1} s^{2} \int_{s^{\tau}}^{\infty} r g(r) d r \leqslant C_{2} s^{2-\tau(c-2)},
\end{aligned}
$$




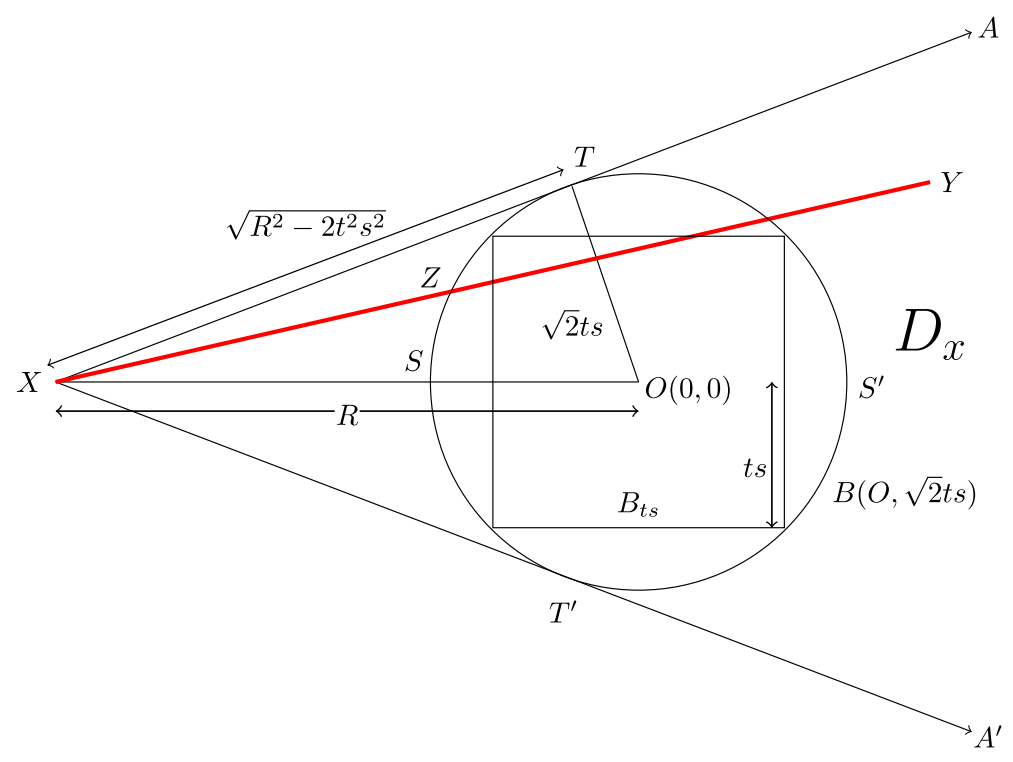

Fig. $4 D_{x}$ is the unbounded region enclosed by the rays $\overrightarrow{T A}$, and $\overrightarrow{T^{\prime} A^{\prime}}$ and the $\operatorname{arc} \overparen{T S^{\prime} T^{\prime}}$

where we have used the fact that the points of $\mathcal{P}_{\lambda}$ from which there is incident on $O$ an edge that is of length longer than $s^{\tau}$ is a Poisson point process of intensity $\lambda g(|x|) 1\left\{x \in B\left(O, s^{\tau}\right)^{c}\right\}$, the inequality $1-e^{-y} \leqslant y$ and the assumption on $g$. Similarly, we can bound the second term on the right-hand side in the last inequality in (2.16) as follows (see Fig. 4),

$$
E\left[\sum_{X, Y \in \mathcal{P}_{\lambda} \cap B(O, \sqrt{2} t s)^{c}} 1\left\{(X, Y) \in \bar{O}_{t, s}\right\}\right]=\lambda^{2} \int_{B(O, \sqrt{2} t s)^{c}} \int_{D_{x} \cap B\left(x, s^{\tau}\right)^{c}} g(|x-y|) d y d x,
$$

where $D_{x}$ is the unbounded region enclosed by the rays $\overrightarrow{T A}$, and $\overrightarrow{T^{\prime} A^{\prime}}$ and the arc $\widehat{T S^{\prime} T^{\prime}}$ as shown in Fig. 4. Changing to polar coordinates and using the obvious bounds for the range of the $y$-variable we can bound the expression on the right in (2.18) by

$$
\begin{aligned}
& C_{3} \int_{\sqrt{2} t s}^{\infty} \int_{0}^{2 \pi} R\left(\int_{s^{\tau} \vee \sqrt{R^{2}-2 t^{2} s^{2}}}^{\infty} r g(r) d r\right) d \phi d R \\
& \leqslant C_{4} \int_{\sqrt{2} t s}^{\infty}\left(s^{\tau} \vee \sqrt{R^{2}-2 t^{2} s^{2}}\right)^{2-c} R d R \\
& =C_{4} \int_{t s}^{\sqrt{2 t^{2} s^{2}+s^{2 \tau}}} s^{\tau(2-c)} R d R+C_{4} \int_{\sqrt{2 t^{2} s^{2}+s^{2 \tau}}}^{\infty}\left(R^{2}-2 t^{2} s^{2}\right)^{\frac{2-c}{2}} R d R \\
& =C_{5} s^{-\tau(c-4)}+C_{6} \int_{s^{\tau}}^{\infty} u^{3-c} d u=C_{7} s^{-\tau(c-4)},
\end{aligned}
$$


where we have used the assumption that $g(r) \leqslant C r^{-c}$. Substituting from (2.17) and (2.19) in (2.16) we obtain

$$
P\left(D_{t s}\left(s^{\tau}\right)\right) \leqslant C_{2} s^{2-\tau(c-2)}+C_{7} s^{-\tau(c-4)} .
$$

Hence, $P\left(D_{t s}\left(s^{\tau}\right)\right) \rightarrow 0$ as $s \rightarrow \infty$, since $\tau>\frac{2}{c-2}$ and $c>4$.

The following corollary gives us the precise form in which we will be using Proposition 2.3. Recall that $A_{2 t s, 4 t s}$ is the annulus $[-4 t s, 4 t s]^{2} \backslash[-2 t s, 2 t s]^{2}$.

Corollary 2.4 For the graph $G_{\lambda}$ with the connection function $g$ satisfying $g(r)=$ $O\left(r^{-c}\right)$ as $r \rightarrow \infty$, let $L_{t s}(\tau)$ be the event that there exists an edge of length longer than $s^{\tau}$ intersecting the annulus $A_{2 t s, 4 t s .}$ Then for any $c>4, t>0$ and $\tau>\frac{2}{c-2}$ we have $P\left(L_{t s}(\tau)\right) \rightarrow 0$ as $s \rightarrow \infty$.

By assumption in part (i) of Theorem 1.2, we have for some $c_{0}>0$,

$$
C_{s}(1) \geqslant c_{0} \text { for all } s \geqslant 1 .
$$

Proposition 2.5 below is a restatement of the first assertion in Theorem 1.2 for the case $\rho=2$. We shall first use this proposition to extend the result for general $\rho$ and then follow it up with the proof of the proposition.

Proposition 2.5 Suppose (2.20) holds for the graph $G_{\lambda}^{e}$ with the connection function $g$ satisfying $g(r)=O\left(r^{-c}\right)$ as $r \rightarrow \infty$ for some $c>4$. Then $\inf _{s \geqslant 1} C_{s}(2)>0$.

Proof of Theorem 1.2 (i) Let $s \geqslant 1$. Assuming that Proposition 2.5 holds, it suffices to prove the result for $\rho>2$. We need to build a left to right crossing in $[0, \rho s] \times$ $[0, s]$. Observe that

$$
[0, \rho s] \times[0, s] \subset \bigcup_{j=0}^{n_{\rho}}((j s, 0)+[0,2 s] \times[0, s]),
$$

where $n_{\rho} \leqslant\lfloor\rho\rfloor$. Let $F_{S}(\rho)$ be the event that there exists left to right crossing in $(j s, 0)+[0,2 s] \times[0, s]$ for all $j=0,1,2, \ldots, n_{\rho}$ and top to down crossing in $(j s, 0)+[0, s] \times[0, s]$ for all $j=1,2, \ldots, n_{\rho}$. From $(2.21)$ we have $F_{S}(\rho) \subset L R_{s}(\rho)$ (see Fig. 5). Using this inclusion and applying the FKG inequality (Theorem 2.1) we obtain

$$
C_{S}(\rho) \geqslant P\left[L R_{S}(2)\right]^{n_{\rho}+1} P\left[T D_{S}(1)\right]^{n_{\rho}} .
$$

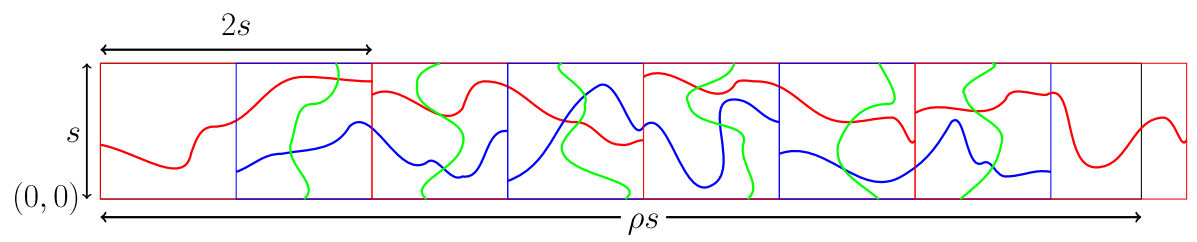

Fig. 5 A realization of the event $F_{S}(\rho)$ 
The assertion in Theorem 1.2 ( $i$ ) now follows from (2.20) and Proposition 2.5. It remains to prove Proposition 2.5 .

We push the technical part of the proof of Proposition 2.5 into Proposition 2.6 that follows from a geometric construction. Given Proposition 2.6 the proof of Proposition 2.5 follows easily.

Definition 2.1 (Circuit) A circuit around $S$ in the region $T \backslash S, S \subset T \subset \mathbb{R}^{2}$ where both $S, T$ are connected subsets, is a path that starts and ends at the same point and is entirely contained inside $T \backslash S$ and goes around $S$. Note that the end points of the edges that contribute to the path may lie outside $T \backslash S$. Recall that $B_{S}:=[-s, s]^{2}$ and for $t>s, A_{s, t}:=B_{t} \backslash B_{s}$. Let $\mathcal{A}_{s}$ be the event that there exists a circuit in the annulus $A_{s, 2 s}$.

Proposition 2.6 Suppose the conditions given in Proposition 2.5 hold. Then there exist constants $c_{2}>0, C>4$ and an increasing sequence of scales $\left\{s_{n}\right\}_{n} \geqslant 1$ satisfying $4 s_{n} \leqslant s_{n+1} \leqslant C s_{n}$ such that $P\left[\mathcal{A}_{s_{n}}\right] \geqslant c_{2}$ for all $n \geqslant 1$.

Proof of Proposition 2.5 Let $\left\{s_{n}\right\}_{n} \geqslant 1$ be the sequence of scales as in Proposition 2.6. For any $s \geqslant 1$ let $k=k(s)$ be such that $s_{k} \leqslant s<s_{k+1}$. Since $s_{k+1} \leqslant C s_{k}$ with $C$ as in Proposition 2.6 we have

$$
[0,2 s] \times\left[0, s_{k}\right] \subset \bigcup_{i=0}^{n_{1}}\left(\left(i s_{k}, 0\right)+\left[0,2 s_{k}\right] \times\left[0, s_{k}\right]\right),
$$

where $n_{1}=\left\lfloor 2 s / s_{k}\right\rfloor+1 \leqslant 2 C+1$.

Let $F_{S}$ be the event (see Fig. 6) that there is a left to right crossing in each of the rectangles $\left(i s_{k}, 0\right)+\left[0,2 s_{k}\right] \times\left[0, s_{k}\right]$ for $i=0,1, \ldots, n_{1}$ and top to down crossing in each of the squares $\left(i s_{k}, 0\right)+\left[0, s_{k}\right] \times\left[0, s_{k}\right]$ for $i=1,2, \ldots, n_{1}$. Clearly

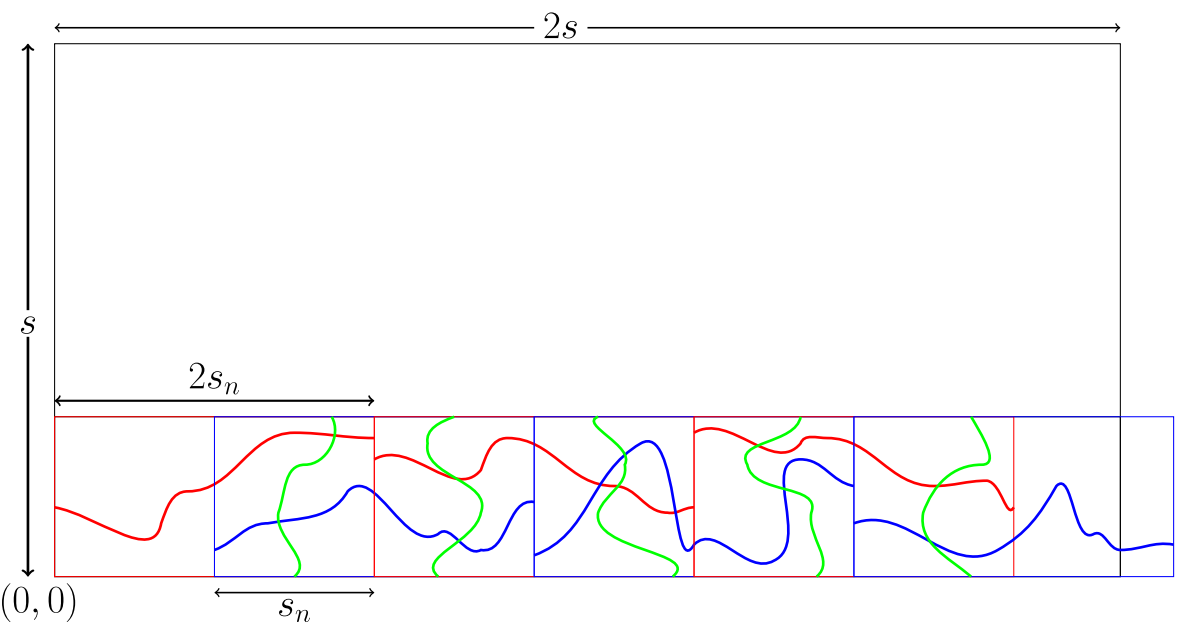

Fig. 6 The event $F_{s}$, occurrence of which gives a left-right crossing in $[0,2 s] \times[0, s]$ 
$F_{s} \subset L R_{s}(2)$ and by Proposition 2.6 we have $C_{s_{k}}(2) \geqslant P\left(\mathcal{A}_{s_{k}}\right) \geqslant c_{2}$. It follows by the FKG inequality (Theorem 2.1) that $C_{s}(2) \geqslant c_{2}^{n_{1}+1} c_{0}^{n_{1}}>0$. This proves Proposition 2.5.

We now proceed to prove Proposition 2.6 where the particular characteristics of the model come into play.

Proof of Proposition 2.6 Fix $s \geqslant 1$. For $\alpha, \beta \in[-s / 2, s / 2], \alpha<\beta$, let $\mathcal{H}_{s}(\alpha, \beta)$ be the event (see Fig. 7) that there exists a path in the box $B_{s / 2}$ from left to $\{s / 2\} \times[\alpha, \beta]$. For $\alpha \in[0, s / 2]$, define $\chi_{s}(\alpha)$ be the event that there exists a path from $\{-s / 2\} \times$ $[-s / 2,-\alpha]$ to $\{s / 2\} \times[\alpha, s / 2]$ and there exists a path from $\{-s / 2\} \times[\alpha, s / 2]$ to $\{s / 2\} \times[-\alpha,-s / 2]$ in $B_{s / 2}$ (see Fig. 8). Let $\mathcal{H}_{s}^{\prime}(\alpha, \beta)$ be the event that there exists a path in the box $B_{s / 2}$ from right to $\{-s / 2\} \times[\alpha, \beta]$ and $T D_{s}^{\prime}(1)$ be the event that there exists a top to down path in the box $B_{s}$. By rotation and reflection invariance the events $\mathcal{H}_{s}(-\alpha,-s / 2), \mathcal{H}_{s}^{\prime}(\alpha,-s / 2), \mathcal{H}_{s}^{\prime}(-\alpha,-s / 2)$ are symmetric versions of $\mathcal{H}_{s}(\alpha, s / 2)$. Observe that

$$
\mathcal{H}_{s}(\alpha, s / 2) \cap \mathcal{H}_{s}(-\alpha,-s / 2) \cap \mathcal{H}_{s}^{\prime}(\alpha, s / 2) \cap \mathcal{H}_{s}^{\prime}(-\alpha,-s / 2) \cap T D_{s / 2}^{\prime}(1) \subset \chi_{s}(\alpha) .
$$

Given the assumption (2.20) that the probability of crossing boxes $C_{S}(1)$ is uniformly bounded away from zero, the following two Lemmas (Lemmas 2.1, 2.2 and 3.1 from [51]) provide lower bounds for probabilities of certain paths that will allow us to glue them together to construct paths with desired properties. These Lemmas

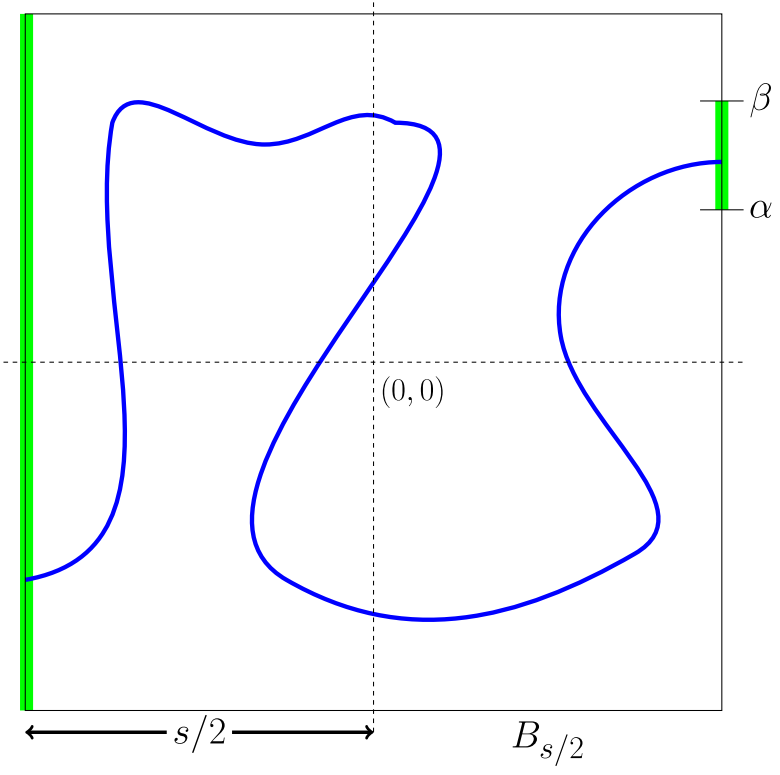

Fig. 7 Event $\mathcal{H}_{s}(\alpha, \beta)$ 


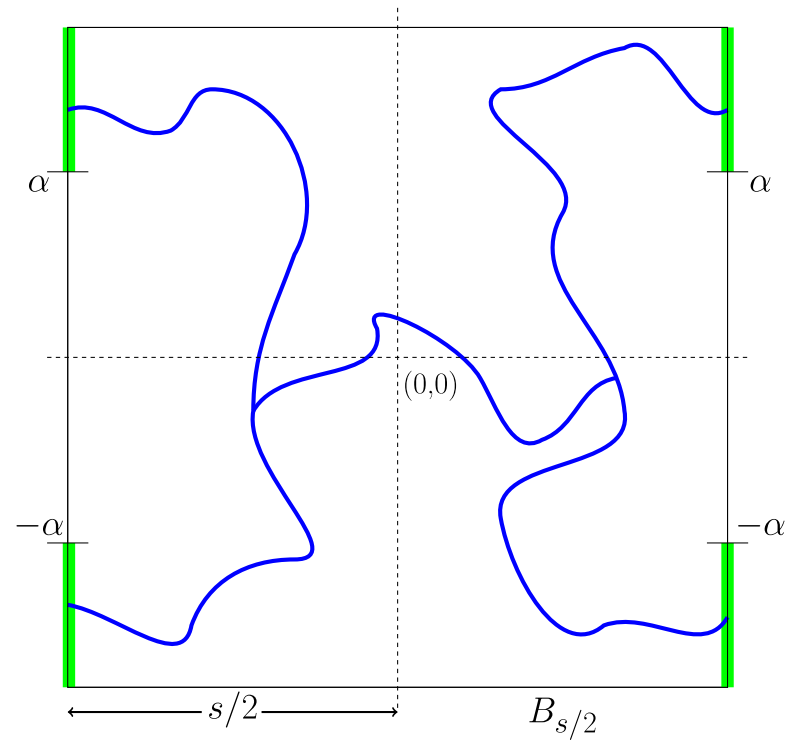

Fig. 8 Event $\chi_{s}(\alpha)$

are true for any planar percolation model which in conjunction with a result such as Corollary 2.4 allows us to derive the RSW result.

Lemma 2.7 If for some $c_{0}>0, \inf _{s} \geqslant 1 C_{s}(1) \geqslant c_{0}$, then for all $s \geqslant 1$ there exists $\alpha_{s} \in[0, s / 4]$ and $c_{1}>0$ such that,

1. for all $0 \leqslant \alpha \leqslant \alpha_{s}$

$$
P\left(\chi_{s}(\alpha)\right) \geqslant c_{1},
$$

2. if $\alpha_{s}<s / 4$, then for all $\alpha_{s} \leqslant \alpha \leqslant s / 2$

$$
P\left(\mathcal{H}_{s}(0, \alpha)\right)-P\left(\mathcal{H}_{s}(\alpha, s / 2)\right) \geqslant c_{0} / 4 \text {. }
$$

Lemma 2.8 Let $\alpha_{s}$ be as in Lemma 2.7. Then the following two statements are true.

(i) There exists $c_{2}>0$ such that whenever $\alpha_{s} \leqslant 2 \alpha_{\frac{2}{3} s}$ for some $s \geqslant 2$, then

$$
P\left(\mathcal{A}_{s}\right) \geqslant c_{2} \text {. }
$$

(ii) For any $s \geqslant 1$ if $P\left(\mathcal{A}_{s}\right) \geqslant c_{2}$ and $\alpha_{t} \leqslant s$ for some $t \geqslant 4 s$, then there exists $c_{3}>0$ such that

$$
P\left(\mathcal{A}_{t}\right) \geqslant c_{3}
$$


We now proceed in a sequential manner to obtain the sequence $\left\{s_{n}\right\}$. Let $\alpha_{s}$ be as in Lemma 2.7. Since $\alpha_{s}<s$, for any $s_{0} \geqslant 1$ there must exist a $s_{1}>s_{0}$ such that

$$
\alpha_{s_{1}} \leqslant 2 \alpha_{\frac{2}{3} s_{1}} .
$$

By (2.26) and Lemma 2.8 (i) we have

$$
P\left(\mathcal{A}_{s_{1}}\right) \geqslant c_{2} .
$$

Having found $s_{1}$ the next task is to find $s_{2}$. This is done using the two steps described in the following Lemma. In order to state the Lemma we need to choose some constants that will be used later.

Let $c_{3}$ be as in Lemma 2.8 (ii) and $c_{0}>0$ be as in (2.20). Since $c_{0}, c_{3} \in(0,1)$ and $c>4$, we can and do choose $C_{1}>16$ such that,

$$
\left(1-\frac{c_{3}}{2}\right)^{\left\lfloor\log _{5} \frac{C_{1}}{2}\right\rfloor-1}<\frac{c_{0}}{4} \text {. }
$$

Pick $\epsilon<\frac{c_{3}}{2}$ such that

$$
\left(\frac{1-\frac{c_{3}}{2}}{1-\epsilon}\right)^{\left\lfloor\log _{5} \frac{C_{1}}{2}\right\rfloor-1}<\frac{c_{0}}{4} .
$$

Fix $\tau \in\left(\frac{2}{c-2}, 1\right)$. This is possible since $c>4$. By Corollary 2.4 there exists $s_{0} \geqslant 1$ such that for all $s \geqslant s_{0}$ we have

$$
P\left(L_{\frac{5^{i}}{4} s}(\tau)\right) \leqslant \frac{c_{3}}{2} \quad \text { for all } i=2,3, \ldots,\left\lfloor\log _{5} \frac{C_{1}}{2}\right\rfloor,
$$

and

$$
P\left(\bigcap_{i=2}^{\left\lfloor\log _{5} \frac{C_{1}}{2}\right\rfloor} L_{\frac{5^{i}}{4} s}^{c}(\tau)\right)>1-\epsilon .
$$

Lemma 2.9 Fix $\tau \in\left(\frac{2}{c-2}, 1\right)$ and let $C_{1}, \epsilon, s_{0}$ satisfy (2.28)-(2.31). If $P\left(\mathcal{A}_{s}\right) \geqslant c_{2}$ for any $s \geqslant s_{0}$, then there exists $s^{\prime} \in\left[4 s, C_{1} s\right]$ such that $\alpha_{s^{\prime}} \geqslant s$. Further, there exists a constant $C_{1}^{\prime}$ and $s_{2} \in\left[s^{\prime}, C_{1}^{\prime} s^{\prime}\right]$ such that $\alpha_{s_{2}} \leqslant 2 \alpha_{\frac{2}{3} s_{2}}$.

The proof of Proposition 2.6 now follows easily from Lemma 2.9. In view of (2.27) there exists by Lemma $2.9 s_{1}^{\prime} \in\left[4 s_{1}, C_{1} s_{1}\right]$ such that $\alpha_{s_{1}^{\prime}} \geqslant s_{1}$. Consequently, by the second assertion of Lemma 2.9 there exists a $C_{1}^{\prime}$ and $s_{2} \in\left[s_{1}^{\prime}, C_{1}^{\prime} s_{1}^{\prime}\right]$ such that $\alpha_{s_{2}} \leqslant 2 \alpha_{\frac{2}{3} s_{2}}$. An application of Lemma $2.8(i)$ now yields

$$
P\left(\mathcal{A}_{s_{2}}\right) \geqslant c_{2} \text {. }
$$

Observe that $4 s_{1} \leqslant s_{1}^{\prime} \leqslant s_{2} \leqslant C_{1}^{\prime} s_{1}^{\prime} \leqslant C_{1} C_{1}^{\prime} s_{1}$. Setting $C=C_{1} C_{1}^{\prime}$ and iterating this procedure we obtain the desired sequence $\left\{s_{n}\right\}_{n} \geqslant 1$. This proves the Proposition 2.6 except for Lemma 2.9 .

Proof of Lemma 2.9 The first part of the proof of Lemma 2.9 is an adaptation of the proof of Lemma 3.2 in [51] (see remark above Theorem 1.2). Suppose that for some 
$s \geqslant s_{0}$ and $c_{2}>0, P\left(\mathcal{A}_{s}\right) \geqslant c_{2}$. Suppose if possible $\alpha_{t}<s$ for all $t \in\left[4 s, C_{1} s\right]$. If we take $t=C_{1} s$, then we have $\alpha_{C_{1} s}<s<\frac{C_{1}}{4} s$. It follows by Lemma 2.7 (ii) that

$$
P\left(\mathcal{H}_{C_{1} s}(0, s)\right)-P\left(\mathcal{H}_{C_{1} s}\left(s, \frac{C_{1} s}{2}\right)\right) \geqslant \frac{c_{0}}{4} .
$$

We will now derive a contradiction to (2.33). Note that $\frac{5^{i} s}{2} \in\left[4 s, C_{1} s\right]$ for $i=$ $2,3, \ldots,\left\lfloor\log _{5} \frac{C_{1}}{2}\right\rfloor$. Since $P\left(\mathcal{A}_{s}\right) \geqslant c_{2}$, taking $t=\frac{5^{i} s}{2}$ we have by Lemma 2.8 (ii)

$$
P\left(\mathcal{A}_{\frac{5 i}{2} s}\right) \geqslant c_{3} \text {. }
$$

Combining (2.34) and (2.30) we can write for $i=2,3, \ldots,\left\lfloor\log _{5} \frac{C_{1}}{2}\right\rfloor$,

$$
P\left(\mathcal{A}_{\frac{5^{i}}{2} s} \cap L_{\frac{5^{i}}{4} s}^{c}(\tau)\right) \geqslant \frac{c_{3}}{2} .
$$

Let $\mathcal{E}_{s}$ be the event that there exists a circuit in $A_{s, \frac{C_{1} s}{2}}$. Let $M=\left\lfloor\log _{5} \frac{C_{1}}{2}\right\rfloor$. Observe that $\mathcal{E}_{s}$ occurs if $\mathcal{A}_{\frac{5}{2}}$ s occurs for some $i=2,3, \ldots, M$. Hence,

$$
P\left(\mathcal{E}_{s}\right) \geqslant P\left(\bigcup_{i=2}^{M} \mathcal{A}_{\frac{5^{i}}{2} s}\right) .
$$

Given the event $\bigcap_{i=2}^{M} L_{\frac{5^{i}}{4} s}^{c}$, the occurrence or otherwise of the event $\mathcal{A}_{\frac{5 i}{2} s}$ depends only on the points of $\mathcal{P}_{\lambda}$ that fall in the region $A_{\frac{5 i}{2} s-s^{\tau}, 5^{i} s+s^{\tau}}$. Since $\tau<1$, $A_{\frac{5^{i}}{2} s-s^{\tau}, 5^{i} s+s^{\tau}} \subset A_{\frac{5^{i}}{2} s-s, 5^{i} s+s}$ and $A_{\frac{5^{i}}{2} s-s, 5^{i} s+s} \cap A_{\frac{5^{i+1}}{2} s-s, 5^{i+1} s+s}=\emptyset$. Consequently the events $\mathcal{A}_{\frac{5}{2}}$ s for $i=2,3, \ldots, M$ are conditionally independent. Using this fact in (2.36) and by substituting from (2.29)-(2.31) and (2.35) yields

$$
\begin{aligned}
P\left(\mathcal{E}_{s}^{c}\right) & \leqslant P\left(\bigcap_{i=2}^{M} \mathcal{A}_{\frac{5^{i}}{2} s}^{c}\right) \\
& \leqslant P\left(\bigcap_{i=2}^{M} \mathcal{A}_{\frac{5^{i}}{2} s}^{c} \mid \bigcap_{i=2}^{M} L_{\frac{5^{i}}{4} s}^{c}(\tau)\right)=\prod_{i=2}^{M} P\left(\mathcal{A}_{\frac{5^{i}}{2} s}^{c} \mid \bigcap_{i=2}^{M} L_{\frac{5^{i}}{4} s}^{c}(\tau)\right) \\
& \leqslant \frac{\prod_{i=2}^{M} P\left(\mathcal{A}_{\frac{5^{i}}{2} s}^{c} \cap L_{\frac{5^{i}}{4} s}^{c}(\tau)\right)}{\left(P\left(\bigcap_{i=2}^{M} L_{\frac{5^{i}}{4} s}^{c}(\tau)\right)\right)^{M-1}} \leqslant \frac{\prod_{i=2}^{M}\left(1-P\left(\mathcal{A}_{\frac{5^{i}}{2} s} \cap L_{\frac{5^{i}}{4} s}^{c}(\tau)\right)\right)}{(1-\epsilon)^{M-1}} \\
& <\frac{c_{0}}{4} .
\end{aligned}
$$

Let $L H_{s}$ be the event that there is a path from left to $\{0\} \times[0, s]$ in $\left(-\frac{C_{1} s}{2}, 0\right)+B_{\frac{C_{1} s}{2}}$ and $U H_{s}$ be the event that there is path from left to $\{0\} \times\left[s, \frac{C_{1} s}{2}\right]$ in $\left(-\frac{C_{1} s}{2}, 0\right)+$ $B_{\frac{C_{1} s}{2}}$. 


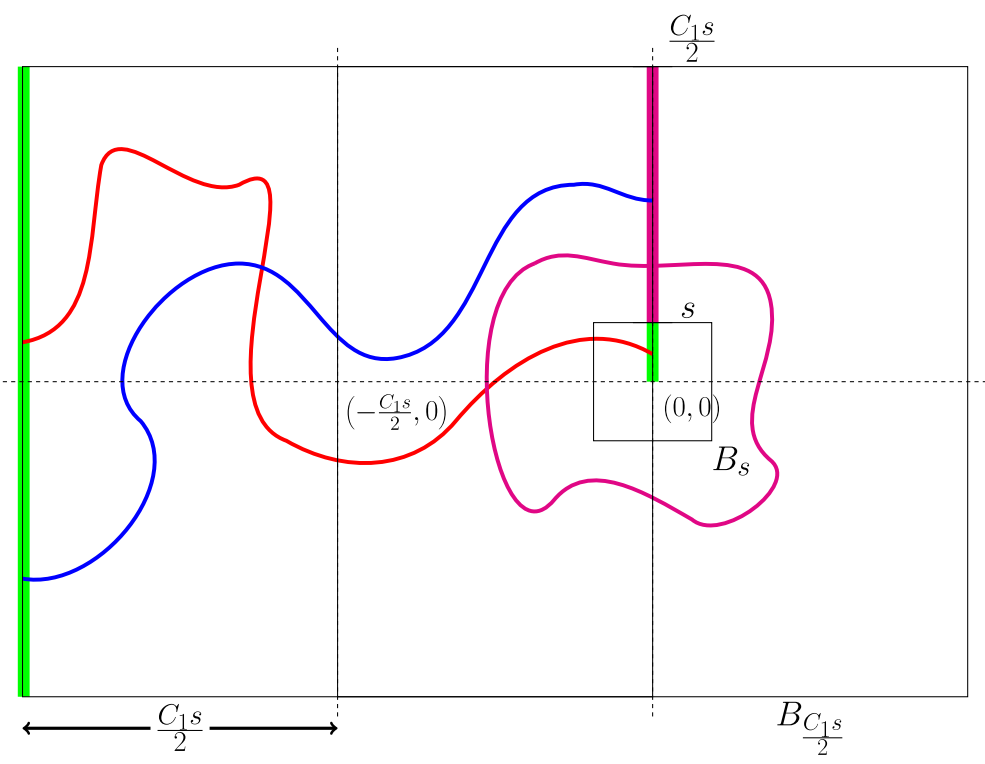

Fig. 9 A realisation of the event $L H_{s}$ (red path), $U H_{s}$ (blue path) and $\mathcal{E}_{s}$ (magenta circuit)

Observe that when $L H_{s} \cap U H_{s}^{c}$ occurs, there cannot exist a circuit in $A_{s, \frac{C_{1} s}{2}}$ around $B_{s}$, that is $L H_{s} \cap U H_{s}^{c} \subset \mathcal{E}_{s}^{c}$ (see Fig. 9). This observation together with (2.37) and translation invariance yields

$$
\begin{aligned}
\frac{c_{0}}{4}>P\left(L H_{s} \cap U H_{s}^{c}\right) & \geqslant P\left(L H_{s}\right)-P\left(U H_{s}\right) \\
& =P\left(\mathcal{H}_{C_{1} s}(0, s)\right)-P\left(\mathcal{H}_{C_{1} s}\left(s, \frac{C_{1} s}{2}\right)\right),
\end{aligned}
$$

which contradicts (2.33). Hence, there must exists a $s^{\prime} \in\left[4 s, C_{1} s\right]$, such that $\alpha_{s^{\prime}} \geqslant s$. This proves the first assertion in Lemma 2.9.

From the first part of this lemma there exists $s^{\prime} \in\left[4 s, C_{1} s\right]$ such that

$$
\alpha_{s^{\prime}} \geqslant s \geqslant \frac{s^{\prime}}{C_{1}} \text {. }
$$

We shall prove the second part as well by contradiction. Suppose if possible $\alpha_{t}>$ $2 \alpha_{2 t / 3}$ for all $t \geqslant s^{\prime}$. By iterating this inequality we obtain

$$
\alpha_{\left(\frac{3}{2}\right)^{k} s^{\prime}}>2 \alpha_{\left(\frac{3}{2}\right)^{k-1}{ }_{s^{\prime}}}>2^{k} \alpha_{s^{\prime}} \geq 2^{k} \frac{s^{\prime}}{C_{1}},
$$

for all $k \geqslant 1$, where the last inequality follows from (2.39). Since $\alpha_{s} \leqslant \frac{s}{4}$ for all $s \geqslant 1$, we have for all $k \geqslant 1$ that

$$
\alpha_{\left(\frac{3}{2}\right)^{k} s^{\prime}} \leqslant \frac{1}{4}\left(\frac{3}{2}\right)^{k} s^{\prime} .
$$


The inequalities (2.40) and (2.41) implies that for all $k \geqslant 1$,

$$
\frac{1}{4}\left(\frac{3}{2}\right)^{k}>\frac{2^{k}}{C_{1}}
$$

which contradicts the fact that $C_{1}<\infty$. Hence, the statement that $\alpha_{t}>2 \alpha_{2 t / 3}$ for

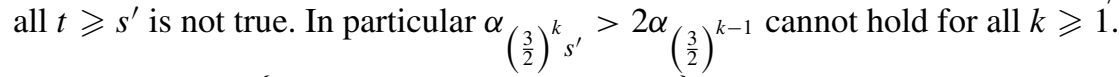

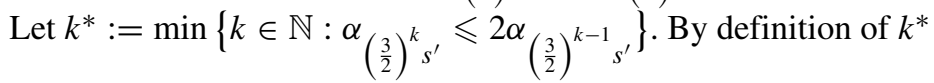

$$
\begin{aligned}
& \alpha_{\left(\frac{3}{2}\right)^{k^{\star}} s^{\prime}} \leqslant 2 \alpha_{\left(\frac{3}{2}\right)^{k^{\star}-1} s^{\prime}} .
\end{aligned}
$$

Again by definition of $k^{*}$ and the argument leading to (2.42) we have $\left(\frac{4}{3}\right)^{k^{\star}-1}<\frac{C_{1}}{4}$, which implies that $k^{\star} \leqslant\left\lfloor\log _{\frac{4}{3}} \frac{C_{1}}{4}\right\rfloor+1$.

Let $s_{2}:=\left(\frac{3}{2}\right)^{k^{\star}} s^{\prime}$. Observe that $s_{2} \geqslant s^{\prime}$ and $s_{2} \leqslant\left(\frac{3}{2}\right)^{\left\lfloor\log _{\frac{4}{3}} \frac{C_{1}}{4}\right\rfloor+1} s^{\prime}$. Let $C_{1}^{\prime}=$ $\left(\frac{3}{2}\right)^{\left.\log _{\frac{4}{3}} \frac{C_{1}}{4}\right\rfloor+1}$. Thus we have found $s_{2} \in\left[s^{\prime}, C_{1}^{\prime} s^{\prime}\right]$ such that $\alpha_{s_{2}} \leqslant 2 \alpha_{\left(\frac{2}{3}\right) s_{2}}$.

This proves second part of Lemma 2.9 and completes the Proof of Proposition 2.6.

\subsection{Proof of Theorem 1.2 (ii)}

As in the proof of the first part it suffices to show the result for $\rho=2$. We first complete the proof using the following lemma which will be proved subsequently using techniques similar to that used to prove Lemma 2.9.

Lemma 2.10 Suppose that $\lim _{s \rightarrow \infty} C_{S}(1)=1$. Then for any fixed $\epsilon>0$ there exists $\eta \in\left(0, \frac{1}{4}\right)$ such that for all s sufficiently large we have

$$
P\left[\text { there exists a circuit around } B_{\eta s} \text { in the annulus } A_{\eta s, \frac{s}{4}}\right]>1-\epsilon .
$$

Let $\eta>0$ be as in Lemma 2.10. Divide the side $\left\{\frac{s}{2}\right\} \times\left[-\frac{s}{2}, \frac{s}{2}\right]$ into intervals labeled $J_{i}(\eta, s), i=1,2, \ldots, \kappa, \kappa=\left\lfloor\frac{1}{2 \eta}\right\rfloor+1$, of length $2 \eta s$ (except for one interval that is of length at most $2 \eta s)$. For $i=1,2, \ldots, \kappa$, let $\mathcal{H}\left(J_{i}(\eta, s)\right)$ be the event that there exists a path in the box $B_{\frac{s}{2}}$ from left to $J_{i}(\eta, s)$. Clearly $L R_{S}(1)=$ $\bigcup_{i=1}^{\kappa} \mathcal{H}\left(J_{i}(\eta, s)\right)$. Using square-root trick (Corollary 2.2) there exists $\beta_{s} \in\left[-\frac{s}{2}, \frac{s}{2}\right]$ satisfying $-\left(\frac{1}{2}-\eta\right) s \leqslant \beta_{s} \leqslant\left(\frac{1}{2}-\eta\right) s$ such that,

$$
\begin{aligned}
P\left(\mathcal{H}_{s}\left(\beta_{s}-\eta s, \beta_{s}+\eta s\right)\right)=\max _{i} P\left(\mathcal{H}\left(J_{i}(\eta, s)\right)\right) & \geqslant 1-\left(1-P\left(\bigcup_{i=1}^{\kappa} \mathcal{H}\left(J_{i}(\eta, s)\right)\right)\right)^{\frac{1}{\kappa}} \\
& =1-\left(1-C_{s}(1)\right)^{\frac{1}{\kappa}}
\end{aligned}
$$




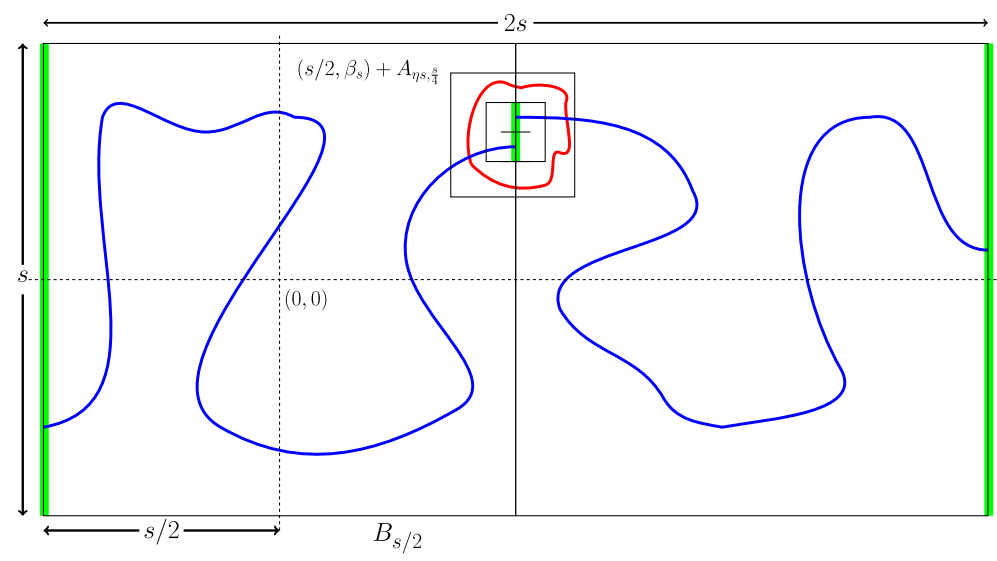

Fig. 10 A realisation of the event $\mathcal{T}_{s}$

Let $R_{S}(\eta), \mathcal{A}_{\eta s, \frac{s}{4}}^{\prime}$ be the events that there exists a path from $\left\{\frac{s}{2}\right\} \times\left[\beta_{s}-\right.$ $\left.\eta s, \beta_{s}+\eta s\right]$ to right in $(s, 0)+B_{\frac{s}{2}}$ and there exists a circuit in $\left(\frac{s}{2}, \beta_{s}\right)+A_{\eta s, \frac{s}{4}}$ respectively (see Fig. 10). By translation and rotation invariance $P\left(R_{S}(\eta)\right) \stackrel{4}{=}$ $P\left(\mathcal{H}_{s}\left(\beta_{s}-\eta s, \beta_{s}+\eta s\right)\right)$. For any $\epsilon>0$, by Lemma 2.10 we have $P\left(\mathcal{A}_{\eta s, \frac{s}{4}}^{\prime}\right)>$ $1-\epsilon$ for all $s$ sufficiently large. Let $\mathcal{T}_{s}:=\mathcal{H}_{s}\left(\beta_{s}-\eta s, \beta_{s}+\eta s\right) \cap R_{s}(\eta) \cap \mathcal{A}_{\eta s, \frac{s}{4}}^{\prime}$. By the FKG inequality and (2.45) we obtain

$$
\begin{aligned}
C_{s}(2) & \geqslant P\left(\mathcal{T}_{s}\right)=P\left(\mathcal{H}_{s}\left(\beta_{s}-\eta s, \beta_{s}+\eta s\right) \cap R_{S}(\eta) \cap \mathcal{A}_{\eta s, \frac{s}{4}}^{\prime}\right) \\
& \geqslant\left[1-\left(1-C_{s}(1)\right)^{\frac{1}{\kappa}}\right]^{2}(1-\epsilon) \rightarrow(1-\epsilon),
\end{aligned}
$$

as $s \rightarrow \infty$. The result now follows since $\epsilon>0$ is arbitrary.

\subsection{Proof of Lemma 2.10}

Fix $\epsilon \in(0,1)$. Since $\lim _{s \rightarrow \infty} C_{s}(1)=1$, there exists a $s_{0}>0$ such that $\inf _{s \geqslant s_{0}} C_{s}(1)>0$ and hence by Theorem $1.2(i) \inf _{s \geqslant s_{0}} C_{s}(4)>0$. By the FKG inequality,

$$
c:=\inf _{s \geqslant s_{0}} P\left(\mathcal{A}_{s}\right) \geqslant \inf _{s \geqslant s_{0}}\left(C_{s}(4)\right)^{4}>0 .
$$

Choose $\eta \in\left(0, \frac{1}{4}\right)$ satisfying

$$
\left(1-\frac{c}{2}\right)^{\left\lfloor\log _{5} \frac{1}{8 \eta}\right\rfloor-1}<\epsilon .
$$

Choose $\delta<\frac{c}{2}$ such that

$$
\left(\frac{1-\frac{c}{2}}{1-\delta}\right)^{\left\lfloor\log _{5} \frac{1}{8 \eta}\right\rfloor-1}<\epsilon
$$


From (2.46) there is a $s_{1}>s_{0}$ such that for all $s \geqslant s_{1}$ and $i=2,3, \ldots,\left\lfloor\log _{5} \frac{1}{8 \eta}\right\rfloor$ we have

$$
P\left(\mathcal{A}_{\frac{5^{i}}{2} \eta s}\right) \geqslant c .
$$

Fix $\tau \in\left(\frac{2}{c-2}, 1\right)$. Such a choice is possible since $c>4$. Using Corollary 2.4 choose $s_{2}>s_{1}$ such that for all $s \geqslant s_{2}$ and $i=2,3, \ldots,\left\lfloor\log _{5} \frac{1}{8 \eta}\right\rfloor$ we have

$$
P\left(L_{\frac{5^{i}}{4} \eta s}(\tau)\right) \leqslant \frac{c}{2}
$$

and

$$
P\left(\bigcap_{i=2}^{\left\lfloor\log _{5} \frac{1}{8 \eta}\right\rfloor} L_{\frac{5^{i}}{4} \eta s}^{c}(\tau)\right)>1-\delta .
$$

Combining (2.49) and (2.50) we can write for $i=2,3, \ldots,\left\lfloor\log _{5} \frac{1}{8 \eta}\right\rfloor$,

$$
P\left(\mathcal{A}_{\frac{5^{i}}{2} \eta s} \cap L_{\frac{5^{i}}{4} \eta s}^{c}(\tau)\right) \geqslant \frac{c}{2} .
$$

Let $\mathcal{E}_{s}$ be the event that there exists a circuit around $B_{\eta s}$ in the annulus $A_{\eta s, \frac{s}{4}}$. Observe that if $\mathcal{A}_{\frac{5 i}{2} \eta s}$ occurs for some $i=2,3, \ldots,\left\lfloor\log _{5} \frac{1}{8 \eta}\right\rfloor$, then $\mathcal{E}_{s}$ will also occur. The result now follows from the same argument as that in Lemma 2.9 below (2.36).

\subsection{Proof of Theorem 1.2 (iii)(Non-percolation at Criticality)}

The proof follows by a renormalization argument that uses the RSW Lemma (Theorem 1.2 (ii)) and Proposition 2.3 on the length of the longest edge in the graph $G_{\lambda}^{e}$. We now define what are known as one arm events that will be useful.

Definition 2.2 (One arm events) Let $S$ be a connected measurable subset of $\mathbb{R}^{2}$. For $A, B \subset S$ with $A, B$ connected and $A \cap B=\phi$,

$A \stackrel{S}{\longleftrightarrow} B:=$ the event that there exists a path in the graph $G_{\lambda}^{e}$ from some point in $A$ to some point in $B$ entirely confined in $S$.

Similarly, for connected subsets $C \subset D \subset \mathbb{R}^{2}$

$C \longleftrightarrow \partial D:=$ the event that there exists a path in the graph $G_{\lambda}^{e}$ from some point in $C$ to some point in $D^{c}$.

Suppose $\theta^{e}(\lambda)>0$, that is, the graph $G_{\lambda}^{e}$ percolates. For $u<n$ and fixed $\tau \in$ $\left(\frac{2}{c-2}, 1\right)$ define the events

$$
E(u, n):=\left\{B_{u} \stackrel{(2 n, 0)+B_{4 n}}{\longleftrightarrow}(4 n, 0)+B_{u}\right\} \text { and } \tilde{E}(u, n):=E(u, n) \cap \mathcal{A}_{u} \cap \tilde{\mathcal{A}}_{u} \cap\left\{M_{4 n} \leqslant n^{\tau}\right\},
$$


where $\mathcal{A}_{u}$ ( $\tilde{\mathcal{A}}_{u}$ resp.) is the event that there exists a circuit around $B_{u}((4 n, 0)+$ $B_{u}$ resp.) in the annulus $A_{u, 2 u}\left((4 n, 0)+A_{u, 2 u}\right.$ resp. $), M_{4 n}:=$ length of the longest edge intersecting the box $(2 n, 0)+B_{4 n}$. We complete the proof using the following proposition, the proof of which shall be provided subsequently.

Proposition 2.11 For the random graph $G_{\lambda}^{e}$ with the connection function $g$ satisfying $g(r)=O\left(r^{-c}\right)$ as $r \rightarrow \infty$ for $c>4$, if $\theta^{e}(\lambda)>0$ then there exists a sequence $\left\{u_{n}\right\}_{n \geqslant 1}$ satisfying $u_{n} \rightarrow \infty$ as $n \rightarrow \infty$ and $u_{n}<\frac{n}{2}$ such that

$$
\lim _{n \rightarrow \infty} P\left(E\left(u_{2 n}, n\right)\right)=1 \text {. }
$$

Let $u_{n}$ be as in Proposition 2.11. We define a coupled nearest neighbor bond percolation model on $4 n \mathbb{Z}^{2}$. The edge $((0,0),(4 n, 0))$ is said to be open if $\tilde{E}\left(u_{2 n}, n\right)$ occurs in $G_{\lambda}^{e}$. An edge that is not open is designated closed. For any two nearest neighbors $z_{1}, z_{2} \in \mathbb{Z}^{2}$ we can define an open edge between $4 n z_{1}, 4 n z_{2}$ in an analogous manner. Otherwise the edge is said to be closed. Denote by $\tilde{G}_{n}$ the graph on the vertex set $4 n \mathbb{Z}^{2}$ formed by the open edges. By translation and rotational invariance, any edge is open with probability $P\left(\tilde{E}\left(u_{2 n}, n\right)\right)$. The edge $((0,0),(4 n, 0))$ being open does not depend on the configuration of points of $\mathcal{P}_{\lambda}$ outside $(2 n, 0)+B_{5 n}$. Thus the status of the edge $((0,0),(4 n, 0))$ can influence that of at most forty neighboring edges. By Theorem 0.0 by Liggett et al. [37] for finitely dependent nearest neighbor bond percolation model on $4 n \mathbb{Z}^{2}, n \in \mathbb{N}$, there exists a constant $q_{0} \in(0,1)$ such that the random graph $\tilde{G}_{n}$ percolates whenever,

$$
P\left(\tilde{E}\left(u_{2 n}, n\right)\right)>q_{0} .
$$

By the FKG inequality $P\left(\mathcal{A}_{s}\right) \geqslant C_{s}(4)^{4}$. Hence, by translational invariance, Theorem $1.2(\mathrm{ii})$, Propositions 2.11 and 2.3 we have

$$
P\left(\tilde{E}\left(u_{2 n}, n\right)\right) \rightarrow 1, \quad \text { as } n \rightarrow \infty .
$$

For $n \in \mathbb{N}$ and $\lambda>0$ define $f_{n}(\lambda):=P\left(\tilde{E}\left(u_{2 n}, n\right)\right)$. Let $X_{h}$ be a Poisson random variable with mean $100 n^{2} h$. Then a simple coupling argument shows that $\mid f_{n}(\lambda+$ $h)-f_{n}(\lambda) \mid \leqslant P\left(X_{h} \geqslant 1\right) \rightarrow 0$ as $h \rightarrow 0$. So $f_{n}$ is continuous.

Let $\Lambda:=\left\{\lambda: \theta^{e}(\lambda)>0\right\}$ be the set of parameters $\lambda>0$ for which $G_{\lambda}^{e}$ percolates. Since $G_{\lambda}^{e}$ percolates if $\tilde{G}_{n}$ does, we have from (2.55) that $\bigcup_{n} f_{n}^{-1}\left(q_{0}, 1\right] \subset \Lambda$. On the other hand if $\lambda \in \Lambda$, then by (2.56) there exists an $n_{0} \in \mathbb{N}$ such that $\lambda \in f_{n_{0}}^{-1}\left(q_{0}, 1\right]$ and hence $\Lambda \subset \bigcup_{n} f_{n}^{-1}\left(q_{0}, 1\right]$. It follows that $\Lambda=\bigcup_{n} f_{n}^{-1}\left(q_{0}, 1\right]$. Since the functions $f_{n}$ are continuous, $\Lambda$ is an open set. This completes the proof of Theorem 1.2 (iii). It remains to prove the Proposition 2.11.

Proof of Proposition 2.11 Since $\theta^{e}(\lambda)>0$, there exists almost surely an infinite connected component in $G_{\lambda}^{e}$. Hence, for any sequence $\left\{u_{n}\right\}_{n} \geqslant 1$ satisfying $u_{n} \rightarrow \infty$ as $n \rightarrow \infty$,

$$
P\left(\mathcal{C} \text { intersects } B_{u_{n}}\right) \rightarrow 1 \text { as } n \rightarrow \infty,
$$


where $\mathcal{C}$ is an infinite connected component in $G_{\lambda}^{e}$. Fix one such sequence for which $u_{n}<\frac{n}{2}$. An immediate consequence of (2.57) is that

$$
P\left(B_{u_{n}} \longleftrightarrow \partial B_{n}\right) \rightarrow 1 \text { as } n \rightarrow \infty .
$$

Let $v_{n}, w_{n}$ be as in Lemma 2.12 below. Define the events

$$
H_{n}:=B_{u_{2 n}} \stackrel{B_{2 n}}{\longleftrightarrow}\{2 n\} \times\left[v_{2 n}, w_{2 n}\right], H_{n}^{\prime}:=(4 n, 0)+B_{u_{2 n}} \stackrel{(4 n, 0)+B_{2 n}}{\longleftrightarrow}\{2 n\} \times\left[v_{2 n}, w_{2 n}\right] .
$$

For $n \in \mathbb{N}$ let $\hat{\mathcal{A}}_{n}$ be the event that there exists a circuit around $\left(2 n, \frac{1}{2}\left(v_{2 n}+w_{2 n}\right)\right)+$ $B_{\frac{1}{2}\left(w_{2 n}-v_{2 n}\right)}$ within the annulus $\left(2 n, \frac{1}{2}\left(v_{2 n}+w_{2 n}\right)\right)+A_{\frac{1}{2}\left(w_{2 n}-v_{2 n}\right),\left(w_{2 n}-v_{2 n}\right)}$. We denote the annulus $\left(2 n, \frac{1}{2}\left(v_{2 n}+w_{2 n}\right)\right)+A_{\frac{1}{2}\left(w_{2 n}-v_{2 n}\right),\left(w_{2 n}-v_{2 n}\right)}$ by $A\left(n, v_{2 n}, w_{2 n}\right)$. By Lemma 2.12 as $n \rightarrow \infty$

$$
P\left(H_{n}\right)=P\left(H_{n}^{\prime}\right) \rightarrow 1 \quad \text { and } \quad A\left(n, v_{2 n}, w_{2 n}\right) \subset(2 n, 0)+B_{2 n} .
$$

By translation invariance, the FKG inequality and Theorem 1.2 ( $i$ i ) we have

$$
\lim _{n \rightarrow \infty} P\left(\hat{\mathcal{A}}_{n}\right)=1 \text {. }
$$

Observe that the paths that enable the events $H_{n}, H_{n}^{\prime}$ must intersect the circuit in $A\left(n, v_{2 n}, w_{2 n}\right)$ (see Fig. 11) and hence

$$
H_{n} \cap H_{n}^{\prime} \cap \hat{\mathcal{A}}_{n} \subset\left\{B_{u_{2 n}} \stackrel{(2 n, 0)+B_{4 n}}{\longleftrightarrow}(4 n, 0)+B_{u_{2 n}}\right\} .
$$

Proposition 2.11 now follows from (2.59)-(2.61).

Lemma 2.12 Consider the random graph $G_{\lambda}^{e}$ with the connection function $g$. If $\theta^{e}(\lambda)>0$, then for any $k \in \mathbb{N}$ there exists sequence $\left\{v_{n}\right\}_{n} \geqslant 1,\left\{w_{n}\right\}_{n} \geqslant 1$ satisfying $\left[v_{2 n}-\frac{1}{2}\left(w_{2 n}-v_{2 n}\right), w_{2 n}+\frac{1}{2}\left(w_{2 n}-v_{2 n}\right)\right] \subset[-2 n, 2 n]$ such that the following holds as $n \rightarrow \infty$

$$
P\left(B_{u_{2 n}} \stackrel{B_{2 n}}{\longleftrightarrow}\{2 n\} \times\left[v_{2 n}, w_{2 n}\right]\right) \rightarrow 1 .
$$

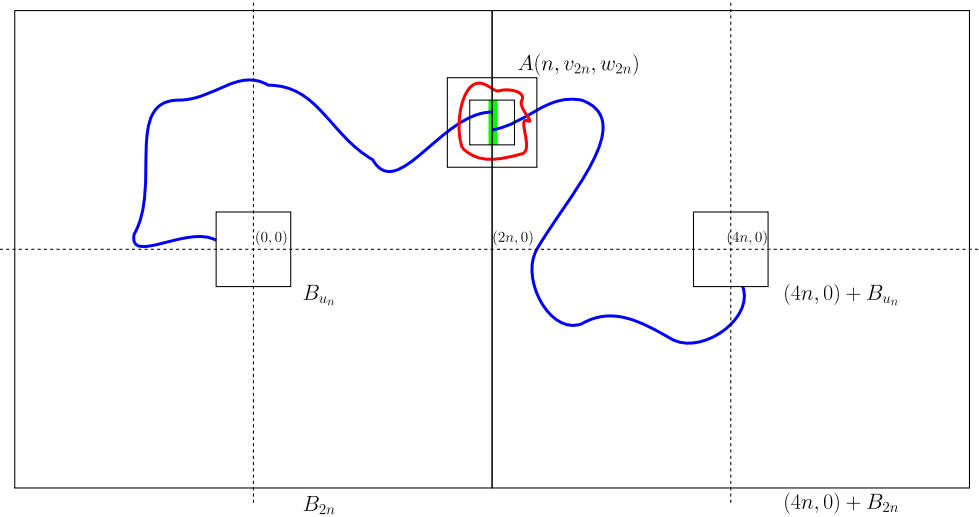

Fig. 11 A realisation of the event $H_{n} \cap H_{n}^{\prime} \cap \hat{\mathcal{A}}_{n}$ 
Proof of Lemma 2.12 Consider the square $B_{2 n}$. By rotational invariance the probability of having a path from $B_{u_{2 n}}$ to any of the eight half intervals on the sides of the square $B_{2 n}$ are same. In other words

$$
P\left(B_{u_{2 n}} \stackrel{B_{2 n}}{\longleftrightarrow} A_{i}\right)=P\left(B_{u_{2 n}} \stackrel{B_{2 n}}{\longleftrightarrow} A_{j}\right),
$$

for $i, j \in\{1,2, \ldots, 8\}$, where $A_{1}=\{2 n\} \times[0,2 n], A_{2}=[0,2 n] \times\{2 n\}, \ldots, A_{8}=$ $\{2 n\} \times[-2 n, 0]$ are the half intervals on the sides of $B_{2 n}$. Applying square-root trick (2.15) and using (2.58) we have

$$
P\left(B_{u_{2 n}} \stackrel{B_{2 n}}{\longleftrightarrow} A_{i}\right) \geqslant 1-\left(1-P\left(B_{u_{2 n}} \longleftrightarrow \partial B_{2 n}\right)\right)^{\frac{1}{8}} \rightarrow 1,
$$

as $n \rightarrow \infty$, for all $i=1, \ldots, 8$. For any $n \in \mathbb{N}$ and $\theta \in[0,2 n]$

$$
\left\{B_{u_{2 n}} \stackrel{B_{2 n}}{\longleftrightarrow}\{2 n\} \times[0, \theta]\right\} \cup\left\{B_{u_{2 n}} \stackrel{B_{2 n}}{\longleftrightarrow}\{2 n\} \times[\theta, 2 n]\right\}=\left\{B_{u_{2 n}} \stackrel{B_{2 n}}{\longleftrightarrow} A_{1}\right\} .
$$

For $\theta \in[0,2 n]$ define the functions

$$
L_{2, n}(\theta):=P\left(\left\{B_{u_{2 n}} \stackrel{B_{2 n}}{\longleftrightarrow}\{2 n\} \times[0, \theta]\right\}\right), U_{2, n}(\theta):=P\left(\left\{B_{u_{2 n}} \stackrel{B_{2 n}}{\longleftrightarrow}\{2 n\} \times[\theta, 2 n]\right\}\right) .
$$

Observe that $U_{2, n}(0)>L_{2, n}(0)=0, L_{2, n}(2 n)>U_{2, n}(2 n)=0, L_{2, n}$ is non-decreasing and $U_{2, n}$ is non-increasing and $L_{2, n}, U_{2, n}$ are continuous. By the properties of $L_{2, n}, U_{2, n}$ there exists $\alpha_{2 n} \in(0,2 n)$ such that $U_{2, n}(\theta) \geqslant L_{2, n}(\theta)$ for $\theta<\alpha_{2 n}, L_{2, n}(\theta) \geqslant U_{2, n}(\theta)$ for $\theta>\alpha_{2 n}$. Further from (2.64) and another application of square-root trick (2.15) we obtain

$$
U_{2, n}\left(\alpha_{2 n}\right)=L_{2, n}\left(\alpha_{2 n}\right) \rightarrow 1 .
$$

Set $\beta_{2 n}=2 n-\alpha_{2 n}>0$ and let $\gamma_{2 n}:=\frac{\alpha_{2 n} \wedge \beta_{2 n}}{16}$. Let $\{2 n\} \times\left[0, \alpha_{2 n}\right]=\bigcup_{j=1}^{k} I_{j}^{(n)}$ where $I_{j}^{(n)}=\{2 n\} \times\left[(j-1) \gamma_{2 n}, j \gamma_{2 n}\right]$, for $j=1,2, \ldots,(k-1)$ and $I_{k}^{(n)}=$ $\{2 n\} \times\left[(k-1) \gamma_{2 n}, k \gamma_{2 n}\right], k=\left\lfloor\frac{\alpha_{2 n}}{\gamma_{2 n}}\right\rfloor+1$ (2.65) together with an application of square-root trick (2.15) yields

$$
\max _{j \in[k]}\left\{P\left(B_{u_{2 n}} \stackrel{B_{2 n}}{\longleftrightarrow} I_{j}^{(n)}\right)\right\} \rightarrow 1 .
$$

as $n \rightarrow \infty$. Let $t(n)=\arg \max _{j \in[k]} P\left(B_{u_{2 n}} \stackrel{B_{2 n}}{\longleftrightarrow} I_{j}^{(n)}\right)$. So (2.62) follows by taking $\left[v_{2 n}, w_{2 n}\right]=I_{t(n)}^{(n)}$.

\section{The Inhomogeneous Enhanced Random Connection Model}

In this section we shall prove the non-trivial phase transition (Theorem 1.1 (ii)), RSW result and non-percolation at criticality (Theorem 1.2) for the inhomogeneous enhanced random connection model. 


\subsection{Proof of Theorem 1.1 (ii)(Non-trivial Phase Transition)}

The proof of the non-trivial phase transition for ieRCM is similar to that of eRCM. The analysis is a bit more involved due to the presence of weights at the vertices. From Theorem $3.2(a 2)$ in [13] with $d=2$ we have that a phase transition occurs in $H_{\lambda}$ provided $\alpha>2, \alpha \beta>4$. However, this condition is required only to show that $\tilde{\lambda}_{c}>0$ while $\tilde{\lambda}_{c}<\infty$ holds over the entire parameter space. Clearly, $H_{\lambda}^{e}$ percolates if $H_{\lambda}$ does and hence $\tilde{\lambda}_{c}^{e}<\infty$.

We now show $\tilde{\lambda}_{c}^{e}>0$ using the same technique as in the proof of Theorem $1.1(i)$. With the same notations as in that proof, we start with the inequality analogous to (2.1),

$$
\tilde{\theta}^{e}(\lambda) \leqslant \sum_{k=0}^{n-1} \sum_{B \in \mathcal{B}_{k}} E^{o}\left[\sum_{\mathbf{x} \in \mathcal{P}_{\lambda, \neq}^{n}} 1\{B(\mathbf{x})=B\}\right] .
$$

Let $\sigma(W):=\sigma\left\{W_{x}: x \in \mathcal{P}_{\lambda}\right\}$ be the sigma algebra generated by the weights. Conditioning on $\mathcal{P}_{\lambda}, \sigma(W)$ and then using (1.1) along with the inequality $1-e^{-x} \leqslant$ $x \wedge 1$ for $x \geqslant 0$ we can bound the expectation inside the sum in (3.1),

$$
\begin{aligned}
& E^{o}\left[\sum_{\mathbf{x} \in \mathcal{P}_{\lambda, \neq}^{n}} 1\{B(\mathbf{x})=B\}\right]=E^{o}\left[\sum_{\mathbf{x} \in \mathcal{P}_{\lambda, \neq}^{n}} E^{o}\left[\prod_{j=1}^{k+1} 1\left\{B_{j}(\mathbf{x})=B_{j}\right\} \mid \mathcal{P}_{\lambda}, \sigma(W)\right]\right] \\
& =E^{o}\left[\sum_{\mathbf{x} \in \mathcal{P}_{\lambda, \neq}^{n}} E^{o}\left[\prod_{j=1}^{k+1} \prod_{l \in B_{j}^{e}} g\left(x_{l-1}, x_{l}\right) 1_{A_{j}}(\mathbf{x}) \mid \mathcal{P}_{\lambda}\right]\right] \\
& \leqslant E^{o}\left[\sum_{\mathbf{x} \in \mathcal{P}_{\lambda, \neq}^{n}} E^{o}\left[\prod_{j=1}^{k+1} \prod_{l \in B_{j}^{e}}\left(\frac{\eta W_{x_{l-1}} W_{x_{l}}}{\left|x_{l}-x_{l-1}\right|^{\alpha}} \wedge 1\right) 1_{A_{j}}(\mathbf{x}) \mid \mathcal{P}_{\lambda}\right]\right] \\
& =E^{o}\left[\sum_{\mathbf{x} \in \mathcal{P}_{\lambda, \neq}^{n}} E^{o}\left[\prod_{\substack{1 \leqslant j \leqslant k+1 \\
j \text { odd }}} \prod_{j}\left(\frac{\eta W_{j}^{e}}{\mid x_{l}-x_{l-1} W_{x_{l}}} \wedge 1\right) 1_{A_{j}}(\mathbf{x}) \prod_{\substack{1 \leqslant j \leqslant k+1 \\
j \text { even }}} \prod_{j}\left(\frac{\eta W_{x_{l-1}} W_{x_{l}}}{\left|x_{l}-x_{l-1}\right|^{\alpha}} \wedge 1\right) 1_{A_{j}}(\mathbf{x}) \mid \mathcal{P}_{\lambda}\right]\right] .
\end{aligned}
$$

By applying the Cauchy-Schwarz inequality followed by using the independence of the weights in the alternating blocks, the conditional expectation on the right-hand side of (3.2) can be bounded from above by

$$
\prod_{j=1}^{k+1} 1_{A_{j}}(\mathbf{x}) \prod_{l \in B_{j}^{e}}\left(E^{o}\left[\left(\frac{\eta W_{x_{l-1}} W_{x_{l}}}{\left|x_{l}-x_{l-1}\right|^{\alpha}}\right)^{2} \wedge 1 \mid \mathcal{P}_{\lambda}\right]\right)^{\frac{1}{2}}
$$


By Lemma 4.3 of [11] we have

$$
\left[E^{o}\left[\left(\frac{W_{1} W_{2}}{t}\right)^{2} \wedge 1\right]\right]^{\frac{1}{2}} \leqslant 1_{\{t<1\}}+c 1_{\{t \geqslant 1\}}(1+(\beta \vee 2) \log t) t^{-\left(\frac{\beta}{2} \wedge 1\right)}=: h(t) \text { (say), }
$$

where $c=\left(1+1_{\{\beta \neq 2\}} \frac{2}{\beta-2}\right)^{\frac{1}{2}}$. Using the bound from (3.4) in (3.3) and then substituting in (3.2) we obtain

$$
E^{o}\left[\sum_{\mathbf{x} \in \mathcal{P}_{\lambda, \neq}^{n}} 1\{B(\mathbf{x})=B\}\right] \leqslant E^{o}\left[\sum_{\mathbf{x} \in \mathcal{P}_{\lambda, \neq}^{n}} \prod_{j=1}^{k+1} 1_{A_{j}}(\mathbf{x}) \prod_{l \in B_{j}^{e}} h\left(\eta^{-1}\left|x_{l}-x_{l-1}\right|^{\alpha}\right)\right] .
$$

By the Campbell-Mecke formula the right-hand side in (3.5) equals

$$
\lambda^{n} \underbrace{\iint \cdots \int}_{n \text { times }} \prod_{j=1}^{k+1} 1_{A_{j}}(\mathbf{x}) \prod_{l \in B_{j}^{e}} h\left(\eta^{-1}\left|x_{l}-x_{l-1}\right|^{\alpha}\right) \prod_{i=1}^{n} d x_{i} .
$$

We evaluate the contribution to (3.6) from blocks of various sizes using calculations similar to those in (2.3)-(2.12). The contribution from a block of size two equals

$$
\int_{\mathbb{R}^{2}} h\left(\eta^{-1}\left|y_{1}-y_{2}\right|^{\alpha}\right) d y_{2}=2 \pi \int_{0}^{\infty} r h\left(\eta^{-1} r^{\alpha}\right) d r .
$$

For $m \geqslant 1$ the contribution from the block of size $2 m+2$ is (see (2.9)) is

$$
2^{m+1} \pi\left(\int_{0}^{\infty} r^{2} h\left(\eta^{-1} r^{\alpha}\right) d r\right)\left(\int_{0}^{\infty} r^{3} h\left(\eta^{-1} r^{\alpha}\right) d r\right)^{m-1}\left(\int_{0}^{\infty} r^{2} h\left(\eta^{-1} r^{\alpha}\right) d r\right) .
$$

Using the contributions from each block $B_{1}, B_{2}, \ldots, B_{k+1}$ from (3.7), (3.8) the expression in (3.6) equals

$$
\left(\prod_{m=0}^{\left\lfloor\frac{n}{2}\right\rfloor}\left(2^{m+1} \pi\right)^{k_{m}}\right)\left(\int_{0}^{\infty} r h\left(\eta^{-1} r^{\alpha}\right) d r\right)^{k_{1}}\left(\int_{0}^{\infty} r^{2} h\left(\eta^{-1} r^{\alpha}\right) d r\right)^{2\left(k-k_{1}\right)}\left(\int_{0}^{\infty} r^{3} h\left(\eta^{-1} r^{\alpha}\right) d r\right)^{\bar{k}},
$$

where $k_{m}$ and $\bar{k}$ are as defined in (2.10) and (2.11). Using (3.6), (3.9) in (3.2) and substituting the resulting expression in (3.1) we obtain

$$
\tilde{\theta}^{e}(\lambda) \leqslant \lambda^{n} \sum_{k=0}^{n-1} \sum_{B \in \mathcal{B}_{k}}\left(\prod_{m=0}^{\left\lfloor\frac{n}{2}\right\rfloor}\left(2^{m+1} \pi\right)^{k_{m}}\right) C_{1}^{4 n}
$$

where $C_{1}=\max \left\{\int_{0}^{\infty} r^{j} h\left(\eta^{-1} r^{\alpha}\right) d r, j=1,2,3\right\}$. By the same arguments as in Theorem 1.1 (i) there exists a constant $C$ such that

$$
\tilde{\theta}^{e}(\lambda) \leqslant(C \lambda)^{n} \rightarrow 0
$$


as $n \rightarrow \infty$ for all $\lambda \in\left(0, \lambda_{0}\right)$, for some $\lambda_{0}>0$ provided $\int_{0}^{\infty} r^{3} h\left(\eta^{-1} r^{\alpha}\right) d r<\infty$, which from (3.4) is true since $\alpha>4$ and $\alpha \beta>8$.

\subsection{Proof of Theorem 1.2 (RSW and Non-percolation at Criticality)}

The RSW results for the ieRCM as enumerated in Theorem 1.2 follow in a manner identical to that for the eRCM once we prove the analog of Proposition 2.3 for the length of the longest edge in the graph $H_{\lambda}$. It thus suffices to prove the following proposition, the proof of which is identical to that of Proposition 2.3 with the obvious changes.

Proposition 3.1 Let $\min \{\alpha, \alpha \beta\}>4$ and consider the graph $H_{\lambda}$ with connection function $g$ as defined in (1.1). For any $s>0$ let $M_{s}$ be the length of the longest edge in $H_{\lambda}$ intersecting the box $B_{s}=[-s, s]^{2}$. Then for any $t>0$ and $\tau>\frac{2}{\min \{\alpha, \alpha \beta\}-2}$ we have $P\left(M_{t s}>s^{\tau}\right) \rightarrow 0$ as $s \rightarrow \infty$.

We shall use the following upper bound on the expected value of the connection function. The proof will be given later. We shall write $E^{W_{x}, W_{y}}$ to denote the expectation with respect to the random weight $W_{x}$ and $W_{y}$.

Lemma 3.2 For $\alpha, \beta, \eta>0$ and any $x \in \mathbb{R}^{2}$ with $|x|^{\alpha}>\eta$ there exists constants $C_{1}, C_{2}, C_{3}$ such that the connection function given by (1.1) satisfies

$$
E^{W_{o}, W_{x}}[g(O, x)]= \begin{cases}C_{1}|x|^{-\alpha}+C_{2}|x|^{-\alpha \beta} \log |x|+C_{3}|x|^{-\alpha \beta}, & \text { if } \beta \neq 1 \\ C_{1}|x|^{-\alpha}(\log |x|)^{2}+C_{2}|x|^{-\alpha} \log |x|+C_{3}|x|^{-\alpha}, & \text { if } \beta=1 .\end{cases}
$$

Proof of Proposition 3.1 Fix $c>4, t>0$. Let $B(O, s):=\left\{x \in \mathbb{R}^{2}:|x| \leqslant s\right\}$ be the ball of radius $s$ centered at the origin. Define the events $D_{s}(l)=\left\{M_{s}>l\right\}$,

$O_{s}(\tau)=\left\{X \in \mathcal{P}_{\lambda}:\right.$ there is an edge of length longer than $s^{\tau}$ incident on $X$ in $\left.H_{\lambda}\right\}$,

$\bar{O}_{t, s}(\tau)=\left\{(X, Y) \in \mathcal{P}_{\lambda}^{2}:\right.$ there is an edge in $H_{\lambda}$ joining $X, Y$ of length longer than $s^{\tau}, \overline{X Y}$ intersects $\left.B(O, \sqrt{2} t s)\right\}$.

Recall that for any two points $x, y \in \mathbb{R}^{2}, \overline{x y}$ denotes the line segment joining $x$ and $y$. Then,

$$
\begin{aligned}
P\left(D_{t s}\left(s^{\tau}\right)\right) & \leqslant E\left[\sum_{X, Y \in \mathcal{P}_{\lambda}} 1\left\{\overline{X Y} \text { intersects } B_{t s}\right\} 1\left\{|X-Y| \geqslant s^{\tau}\right\}\right] \\
& \leqslant E\left[\sum_{X \in \mathcal{P}_{\lambda} \cap B(O, \sqrt{2} t s)} 1\left\{X \in O_{s}(\tau)\right\}\right]+E\left[\sum_{X, Y \in \mathcal{P}_{\lambda} \cap B(O, \sqrt{2} t s)^{c}} 1\left\{(X, Y) \in \bar{O}_{t, s}\right\}\right] .
\end{aligned}
$$


In what follows we shall write $E^{W_{x}}$ to denote the expectation with respect to the random weight $W_{x}$. By the Campbell-Mecke formula applied to the first term on the right-hand side of the last inequality in (3.12) we obtain

$$
\begin{aligned}
& E\left[\sum_{X \in \mathcal{P}_{\lambda} \cap B(O, \sqrt{2} t s)} 1\left\{X \in O_{s}(\tau)\right\}\right] \\
& =C \lambda(t s)^{2} E^{W_{o}}\left[P^{o}\left(O \in O_{s}(\tau) \mid W_{o}\right)\right] \\
& =C \lambda(t s)^{2} E^{W_{0}}\left[1-P^{o}\left(\text { none of the edges incident on O is of length } \geqslant s^{\tau} \mid W_{o}\right)\right] \\
& =C \lambda(t s)^{2} E^{W_{0}}\left[1-\exp \left(-\lambda \int_{B\left(O, s^{\tau}\right)^{c}} E^{W_{x}}\left[g(|x|) \mid W_{o}\right] d x\right)\right] \\
& \leqslant C(\lambda t s)^{2} E^{W_{0}}\left[\int_{B\left(O, s^{\tau}\right)^{c}} E^{W_{x}}\left[g(|x|) \mid W_{o}\right] d x\right] \\
& \leqslant C(\lambda t s)^{2} \int_{B\left(O, s^{\tau}\right)^{c}} E^{W_{o}, W_{x}}\left[1-\exp \left(-\frac{\eta W_{o} W_{x}}{|x|^{\alpha}}\right)\right] d x
\end{aligned}
$$

where we have used the fact that conditional on the weight $W_{o}$ at the origin $O$, the points of $\mathcal{P}_{\lambda}$ from which there is incident on $O$ an edge that is of length longer than $s^{\tau}$ is a Poisson point process of intensity $\lambda E^{W_{x}}\left[g(|x|) \mid W_{o}\right] 1\{x \in$ $\left.B\left(O, s^{\tau}\right)^{c}\right\}$ and the inequality $1-e^{-y} \leqslant y$. Using Lemma 3.2 and the fact that $\log r \leqslant r^{\epsilon}$,

$$
\begin{aligned}
\int_{B\left(O, s^{\tau}\right)^{c}} E^{W_{o}, W_{x}}\left[1-\exp \left(-\frac{\eta W_{o} W_{x}}{|x|^{\alpha}}\right)\right] d x & =C_{1} s^{2} \int_{s^{\tau}}^{\infty} r\left(C_{1} r^{-\alpha}+C_{2} r^{-\alpha \beta} \log r+C_{3} r^{-\alpha \beta}\right) d r \\
& \leqslant C_{4} s^{2-\tau(\alpha-2)}+C_{5} s^{2-\tau(\alpha \beta-2)}+C_{6} s^{2-\tau(\alpha \beta-\epsilon-2)} .
\end{aligned}
$$

Similarly, we can bound the second term on the right-hand side in the last inequality in (3.12) as follows (see Fig. 4). Let $\tilde{g}(x, y):=E[g(x, y)], x, y \in \mathbb{R}^{2}$, where $g$ is as specified in (1.1). Observe that $\tilde{g}(x, y)$ depends on $x, y$ only via $|x-y|$ and so by an abuse of notation we will write $\tilde{g}(|x-y|)$ for $\tilde{g}(x, y)$. Then,

$$
\begin{aligned}
E\left[\sum_{X, Y \in \mathcal{P}_{\lambda} \cap B(O, \sqrt{2} t s)^{c}} 1\left\{(X, Y) \in \bar{O}_{t, s}\right\}\right] & \leqslant \lambda^{2} \int_{B(O, \sqrt{2} t s)^{c}} \int_{D_{x} \cap B\left(x, s^{\tau}\right)^{c}} E^{W_{x}, W_{y}}[g(x, y)] d y d x \\
& =\lambda^{2} \int_{B(O, \sqrt{2} t s)^{c}} \int_{D_{x} \cap B\left(x, s^{\tau}\right)^{c}} \tilde{g}(|x-y|) d y d x,
\end{aligned}
$$

where $D_{x}$ is the unbounded region enclosed by the rays $\overrightarrow{T A}$, and $\overrightarrow{T^{\prime} A^{\prime}}$ and the arc $\widehat{T S^{\prime} T^{\prime}}$ as shown in Fig. 4. Changing to polar coordinates as in the proof 
of Proposition 2.3 and using Lemma 3.2 we can bound the last expression in (3.15) by

$$
\begin{aligned}
& C_{7} \int_{\sqrt{2} t s}^{\infty} \int_{0}^{2 \pi} R\left(\int_{\left(s^{\tau} \vee \sqrt{R^{2}-2 t^{2} s^{2}}\right)}^{\infty} r \tilde{g}(r) d r\right) d \phi d R \\
& \leqslant C_{8} \int_{\sqrt{2} t s}^{\infty} \int_{\left(s^{\tau} \vee \sqrt{R^{2}-2 t^{2} s^{2}}\right)}^{\infty} R r\left(r^{-\alpha}+r^{-\alpha \beta} \log r+r^{-\alpha \beta}\right) d r d R \\
& \leqslant C_{9} \int_{\sqrt{2} t s}^{\infty}\left(\left(s^{\tau} \vee \sqrt{R^{2}-2 t^{2} s^{2}}\right)^{2-\alpha}+\left(s^{\tau} \vee \sqrt{R^{2}-2 t^{2} s^{2}}\right)^{2-\alpha \beta}\right) R d R .
\end{aligned}
$$

The integral of the first integrand on righ-hand side in (3.16) can be evaluated as follows,

$$
\begin{aligned}
\int_{\sqrt{2} t s}^{\infty}\left(s^{\tau} \vee \sqrt{R^{2}-2 t^{2} s^{2}}\right)^{2-\alpha} R d R & =\int_{\sqrt{2} t s}^{\sqrt{2 t^{2} s^{2}+s^{2 \tau}}} s^{\tau(2-\alpha)} R d R+\int_{\sqrt{2 t^{2} s^{2}+s^{2 \tau}}}^{\infty}\left(R^{2}-2 t^{2} s^{2}\right)^{\frac{2-\alpha}{2}} R d R \\
& =C_{10} s^{-\tau(\alpha-4)} .
\end{aligned}
$$

Similarly, the second term on the right-hand side in (3.16) can be evaluated to obtain

$$
\int_{\sqrt{2} t s}^{\infty}\left(s^{\tau} \vee \sqrt{R^{2}-2 t^{2} s^{2}}\right)^{2-\alpha \beta} R d R=C_{11} s^{-\tau(\alpha \beta-4)} .
$$

Substituting the resulting term in (3.12) obtained by using (3.17), (3.18) and (3.16) in (3.15) and using (3.14) in (3.13) we obtain

$$
P\left(D_{t s}\left(s^{\tau}\right)\right) \leqslant C_{12}\left(s^{2-\tau(\alpha-2)}+s^{2-\tau(\alpha \beta-2)}+s^{-\tau(\alpha-4)}+s^{-\tau(\alpha \beta-4)}\right) \rightarrow 0,
$$

as $s \rightarrow \infty$ since $\tau>\frac{2}{\min \{\alpha, \alpha \beta\}-2}$ and $\min \{\alpha, \alpha \beta\}>4$.

Proof of Lemma 3.2 We will prove the result for the case $\beta \neq 1$. The proof for $\beta=1$ follows with minor changes. Fix $x \in \mathbb{R}^{2}$ such that $|x|^{\alpha}>\eta$. Then

$$
\begin{aligned}
E^{W_{o}, W_{x}}[g(O, x)] & =E^{W_{o}, W_{x}}\left[1-\exp \left(-\frac{\eta W_{0} W_{x}}{|x|^{\alpha}}\right)\right] \\
& \leqslant E^{W_{o}, W_{x}}\left[\frac{\eta W_{0} W_{x}}{|x|^{\alpha}} \wedge 1\right] \\
& =E^{W_{o}, W_{x}}\left[\frac{\eta W_{0} W_{x}}{|x|^{\alpha}} ; W_{0} W_{x}<\frac{|x|^{\alpha}}{\eta}\right]+P\left(W_{0} W_{x} \geqslant \frac{|x|^{\alpha}}{\eta}\right) .
\end{aligned}
$$

By our assumption on the distribution of the weights it is easy to see that the product $W_{0} W_{x}$ has a density given by

$$
f(w)=\beta^{2} w^{-\beta-1} \log w, w \geqslant 1 .
$$

The first term on the right in (3.19) can be evaluated using the expression in (3.20) and the integration by parts formula to yield 


$$
\begin{aligned}
E^{W_{o}, W_{x}}\left[\frac{\eta W_{0} W_{x}}{|x|^{\alpha}} ; W_{0} W_{x}<\frac{|x|^{\alpha}}{\eta}\right] & =\frac{\eta}{|x|^{\alpha}} \int_{1}^{|x|^{\alpha} / \eta} w f(w) d w \\
& \leqslant c_{1}|x|^{-\alpha}+c_{2}|x|^{-\alpha \beta} \log |x|+c_{3}|x|^{-\alpha \beta},
\end{aligned}
$$

for some constants $c_{1}, c_{2}, c_{3}$. The second term on the right in (3.19) can be computed in a similar fashion,

$$
\begin{aligned}
P\left(W_{0} W_{x} \geqslant \frac{|x|^{\alpha}}{\eta}\right) & =\int_{|x|^{\alpha} / \eta}^{\infty} f(w) d w \\
& \leqslant c_{4}|x|^{-\alpha \beta}+c_{5}|x|^{-\alpha \beta} \log |x|,
\end{aligned}
$$

for some constants $c_{4}, c_{5}$. The assertion (3.11) now follows by substituting from (3.21), (3.22) in (3.19).

\section{The Poisson Stick Model}

\subsection{Proof of Theorem 1.1 (iii) (Non-trivial Phase Transition)}

To show that $\lambda_{P S}<\infty$ consider the graph $P S_{\lambda}$ with half-length density $h$ satisfying $0<\int_{0}^{\infty} \ell^{2} h(\ell) d \ell<\infty$. Let $R_{0}:=\inf \{\ell: h(\ell)>0\}$. Pick any $R_{1}>R_{0}$ finite such that $\int_{R_{0}}^{R_{1}} h(\ell) d \ell>0$. Consider the graph $P S_{\lambda}$ and set sticks of length greater than $2 R_{1}$ to be equal to $2 R_{1}$ to obtain the graph $\tilde{P S} S_{\lambda}$. We have from [46] that the critical threshold parameter $\tilde{\lambda}_{P S}<\infty$ for the truncated model. Since $P S_{\lambda}$ percolates if $\tilde{P S} S_{\lambda}$ does, we have $\lambda_{P S}<\infty$.

The proof of $\tilde{\lambda}_{P S}>0$ is simpler for the Poisson stick model since for each ordered sequence $\mathbf{x}=\left(x_{1}, x_{2}, \ldots, x_{n}\right)$, there is only way in which a path can occur (see Fig. 12). For $x \in \mathcal{P}_{\lambda}$ let $L(x)$ denote the stick centered at $x$. We will denote $L\left(x_{j}\right)$ by $L_{j}$. Then

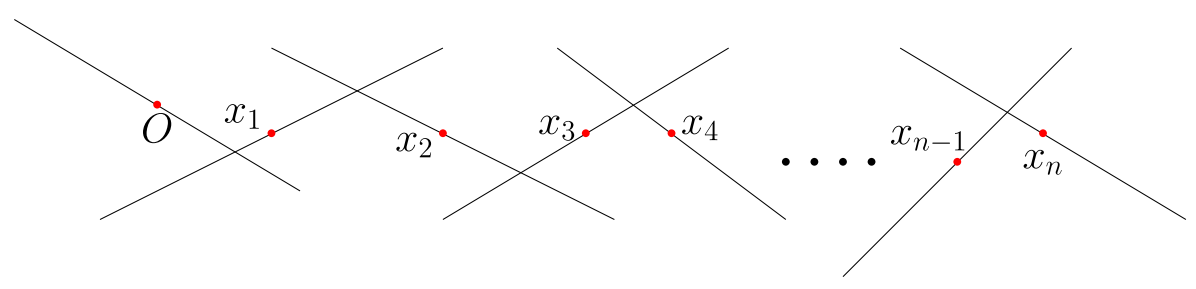

Fig. 12 A self-avoiding path in $P S_{\lambda}$ 
$\theta_{P S}(\lambda) \leqslant P^{o}$ (there is a self-avoiding path on $n$ vertices in $P S_{\lambda}$ starting from $O$ )

$$
\begin{aligned}
& \leqslant E^{o}\left[\sum_{\mathbf{x} \in \mathcal{P}_{\lambda, \neq}^{n}} 1\left\{O \rightarrow x_{1} \rightarrow x_{2} \rightarrow \cdots \rightarrow x_{n} \text { occurs }\right\}\right] \\
& =E^{o}\left[\sum_{\mathbf{x} \in \mathcal{P}_{\lambda, \neq}^{n}} \prod_{j=1}^{n} 1\left\{L_{j} \text { intersects } L_{j-1}\right\}\right] .
\end{aligned}
$$

Sticks $L_{j-1}, L_{j}$ with respective half-lengths $\ell_{j-1}, \ell_{j}$ and orientations $\theta_{j-1}, \theta_{j}$ intersect provided the midpoint $x_{j}$ of $L_{j}$ falls inside a parallelogram with side lengths $2 \ell_{j-1}$ and $2 \ell_{j}$. Such a parallelogram has maximum area $4 \ell_{j-1} \ell_{j}$. By applying the Campbell-Mecke formula to the expectation in (4.1) and using the above observation we obtain

$$
\begin{aligned}
& \theta_{P S}(\lambda) \leqslant \lambda^{n} \underbrace{\int_{0}^{\infty} \cdots \int_{0}^{\infty}}_{(n+1) \text { times }} h\left(\ell_{0}\right) \prod_{j=1}^{n} 4 \ell_{j-1} \ell_{j} h\left(\ell_{j}\right) \prod_{j=0}^{n} d \ell_{j} \\
& =4^{n} \lambda^{n}\left(\int_{0}^{\infty} \ell h(\ell) d \ell\right)^{2}\left(\int_{0}^{\infty} \ell^{2} h(\ell) d \ell\right)^{n-1} \rightarrow 0,
\end{aligned}
$$

as $n \rightarrow \infty$ for all $\lambda>0$ sufficiently small provided $\int_{0}^{\infty} \ell^{2} h(\ell) d \ell<\infty$. This completes the proof of the Theorem 1.1 ( $i i i$ ).

\subsection{Proof of Theorem 1.2 (RSW and Non-percolation at Criticality)}

The proof follows along the same lines as that for the eRCM using the following Proposition on the length of the longest stick in $P S_{\lambda}$. Since $c>3$ we can choose a $\tau<1$ so that the conclusion of Proposition 4.1 holds, which gives us the condition under which the rest of the proof works.

Proposition 4.1 Consider the graph $P S_{\lambda}$ with stick's half-length density function $h$ satisfying $h(\ell)=O\left(\ell^{-c}\right)$ as $\ell \rightarrow \infty$ for some $c>3$. For any $s>0$ let $\bar{M}_{s}$ be the half-length of the longest stick in $P S_{\lambda}$ intersecting the box $B_{s}=[-s, s]^{2}$. Then for any $t>0$ and $\tau>\frac{2}{c-1}$ we have $P\left(\bar{M}_{t s}>s^{\tau}\right) \rightarrow 0$ as $s \rightarrow \infty$.

Proof of Proposition 4.1 Fix $c>3, t>0$ and $\tau>\frac{2}{c-1}$. Recall that $B(O, s)$ denotes the ball of radius $s$ centered at the origin and for $X \in \mathcal{P}_{\lambda}, L_{X}$ denotes the stick with mid point at $X$ with half-length random variable distributed independently according to the probability density function $h$. Define the events $\bar{D}_{s}(\ell)=\left\{\bar{M}_{s}>\ell\right\}$, 
$O_{s}(\tau)=\left\{X \in \mathcal{P}_{\lambda}: L_{X}\right.$ has half-length longer than $s^{\tau}$ and intersects $\left.B(O, \sqrt{2} t s)\right\}$.

Then,

$$
\begin{aligned}
P\left(\bar{D}_{t s}\left(s^{\tau}\right)\right) & \leqslant E\left[\sum_{X \in \mathcal{P}_{\lambda}} 1\left\{L_{X} \text { intersects } B_{t s}\right\} 1\left\{L_{X} \geqslant s^{\tau}\right\}\right] \\
& \leqslant E\left[\sum_{X \in \mathcal{P}_{\lambda} \cap B(O, \sqrt{2} t s)} 1\left\{X \in O_{s}(\tau)\right\}\right]+E\left[\sum_{X \in \mathcal{P}_{\lambda} \cap B(O, \sqrt{2} t s)^{c}} 1\left\{X \in O_{t, s}(\tau)\right\}\right] .
\end{aligned}
$$

By our assumption $h(\ell) \leqslant C \ell^{-c}$ for all $\ell$ sufficiently large. The Campbell-Mecke formula applied to the first term on the right-hand side of the last inequality in (4.2) yields

$$
\begin{aligned}
& E\left[\sum_{X \in \mathcal{P}_{\lambda} \cap B(O, \sqrt{2} t s)} 1\left\{X \in O_{S}(\tau)\right\}\right]=C_{0} \lambda(t s)^{2} P^{o}\left(L_{O} \geqslant s^{\tau}\right) \\
& =C_{1} s^{2} \int_{s^{\tau}}^{\infty} \int_{0}^{\pi} h(\ell) d F(\theta) d \ell=C_{2} s^{2} \int_{s^{\tau}}^{\infty} h(\ell) d \ell \leqslant C_{3} s^{2-\tau(c-1)},
\end{aligned}
$$

for all $s$ sufficiently large. For the second term in (4.2) using the assumption on $h$, the Campbell-Mecke formula and the fact that $|\overline{X Y}| \geqslant s^{\tau} \vee(R-\sqrt{2} t s)$, where $R=|\overline{O X}|$ (see Fig. 13) we obtain for all $s$ sufficiently large

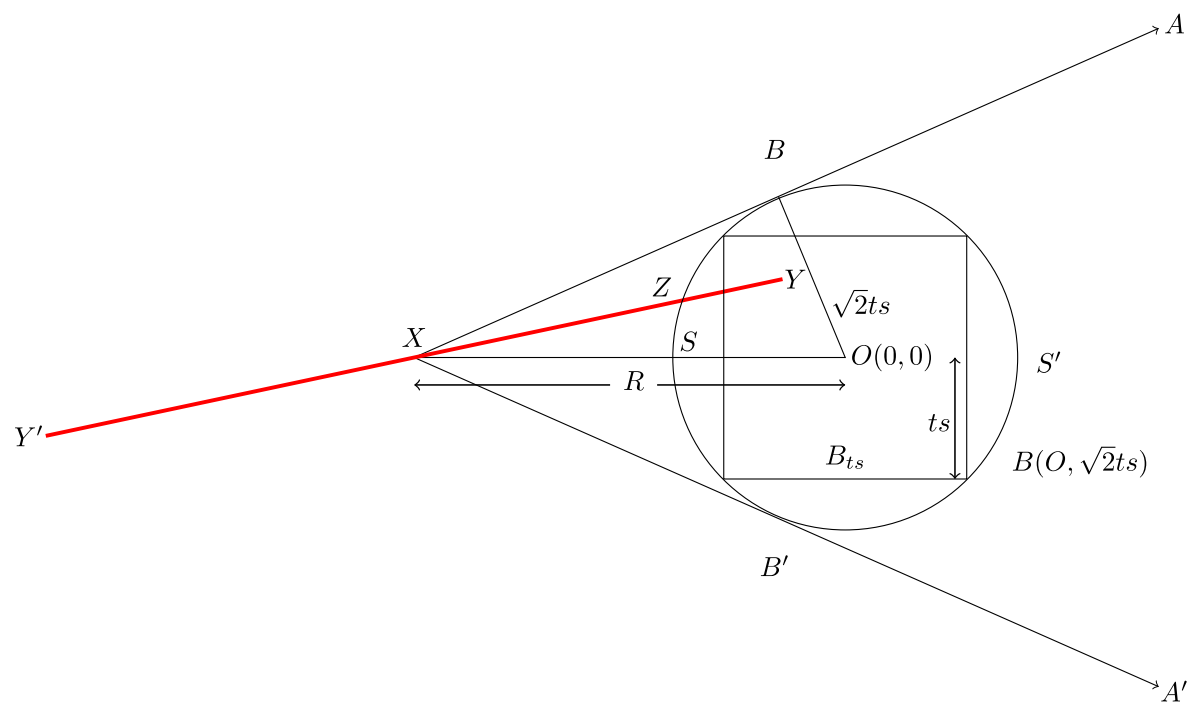

Fig. 13 The red stick with mid point $\mathrm{X}$ intersecting the ball $B(O, \sqrt{2} t s)$ and $R=|\overline{O X}|>\sqrt{2} t s$ 


$$
\begin{aligned}
E\left[\sum_{X \in \mathcal{P}_{\lambda} \cap B(O, \sqrt{2} t s)^{c}} 1\left\{X \in O_{t, s}(\tau)\right\}\right] & \leqslant C \lambda \int_{\sqrt{2} t s}^{\infty} R\left(\int_{s^{\tau} \vee(R-\sqrt{2} t s)}^{\infty} \int_{0}^{\pi} h(\ell) d F(\theta) d \ell\right) d R \\
& =C_{1} \int_{\sqrt{2} t s}^{\infty}\left(s^{\tau} \vee(R-\sqrt{2} t s)\right)^{1-c} R d R \\
& =C_{2} \int_{\sqrt{2} t s}^{\sqrt{2} t s+s^{\tau}} s^{\tau(1-c)} R d R+C_{3} \int_{\sqrt{2} t s+s^{\tau}}^{\infty}(R-\sqrt{2} t s)^{1-c} R d R \\
& =C_{4} s^{-\tau(c-3)}+C_{5} s^{-(\tau(c-2)-1)}+C_{6} \int_{s^{\tau}}^{\infty} u^{1-c}(u+\sqrt{2} t s) d u \\
& =C_{7} s^{-\tau(c-3)}+C_{8} s^{-(\tau(c-2)-1)} .
\end{aligned}
$$

Substituting from (4.3) and (4.4) in (4.2) we obtain

$$
P\left(\bar{D}_{t s}\left(s^{\tau}\right)\right) \leqslant c_{1} s^{-(\tau(c-1)-2)}+c_{2} s^{-\tau(c-3)}+c_{3} s^{-(\tau(c-2)-1)} \rightarrow 0,
$$

as $s \rightarrow \infty$, since $\tau>\frac{2}{c-1}>\frac{1}{c-2}$ for $c>3$.

\section{Appendix}

We need the FKG inequality for functionals on random geometric graphs that depend on the edges that intersect a finite box. The edges in the graph depend not only on the random distribution of points but require some additional randomness to obtain a realization of the edges given the vertex set. The proof thus requires the use of the FKG inequality in the discrete setting as well that for functionals on Poisson point processes. We shall derive the result for the random connection model. Proofs for the other models follow by the same arguments. To preserve monotonicity of our functionals we will need to use a construction of the random connection model from Penrose [41].

We opt to define the random connection model in a more general setting of an arbitrary metric space $(\mathbb{X}, d)$, where $d(\cdot, \cdot)$ is a metric on $\mathbb{X}$. Let $\mathcal{F}$ be a sigma algebra on $\mathbb{X}$ and $S(\mathbb{X})$ be the collection of all locally finite subsets of $\mathbb{X}$. The space $S(\mathbb{X})$ is equipped with the smallest sigma algebra $\mathcal{S}(\mathbb{X})$ containing sets of the form $\{\mathcal{X}$ : $\mathcal{X}(A)=k\}$ for $A \in \mathcal{F}$ and $k \in \mathbb{N}_{0}$, where $\mathcal{X}(A):=\#(\mathcal{X} \cap A)$. For a set $\mathcal{X} \in$ $S(\mathbb{X})$, we attach a sequence $T_{X}=\left(T_{X, i}\right)_{i \in \mathbb{N}} \in[0,1]^{\mathbb{N} \times \mathbb{N}}$ to each point $X \in \mathcal{X}$. For notational simplicity we write $\mathbb{M}$ for $[0,1]^{\mathbb{N} \times \mathbb{N}}$. We denote the marked point set by $\tilde{\mathcal{X}}:=\left\{\left(X, T_{X}\right): X \in \mathcal{X}\right\}$. We shall re-order the points in $\tilde{\mathcal{X}}$ in the increasing order of the first co-ordinate of the marks and re-write the set as $\tilde{\mathcal{X}}:=\left\{\left(X_{i}, T_{i}\right)\right\}_{1 \leqslant i \leqslant \# \mathcal{X}}$, where $T_{i, 1} \leqslant T_{i+1,1}$ for all $1 \leqslant i \leqslant \# \mathcal{X}$. The construction of the graph is a two step procedure. The point set is generated by a marked Poisson point process that will be described below. Given a point set $\tilde{\mathcal{X}}$ the edge set of the graph is defined as follows.

Let $g: \mathbb{X} \times \mathbb{X} \rightarrow[0,1]$ be a non-increasing function. In our case $g(x, y)$ is a function of $d(x, y)$ and so we write $g(d(x, y))$. We say that there is an edge between two points $X_{i}, X_{j} \in \mathcal{X}$ with $i<j$, if $T_{i, j}<g\left(d\left(X_{i}, X_{j}\right)\right)$. We denote the resultant graph by $G(\tilde{\mathcal{X}}, g)$. 
We shall use the following coupling to claim that certain add-one cost functionals are non-negative which will be required to apply the FKG inequality for functionals of a Poisson point process. For $\left(x, T_{x}\right) \in \mathbb{X} \times \mathbb{M}$, let $G_{x}\left(\tilde{\mathcal{X}} \cup\left\{\left(x, T_{x}\right)\right\}, g\right)$ be the random graph on the point set $\tilde{\mathcal{X}}$ obtained by deleting the vertex $x$ and all the edges incident on $x$ from the graph $G\left(\tilde{\mathcal{X}} \cup\left\{\left(x, T_{x}\right)\right\}, g\right)$.

We denote the set of all graphs on the set of point sets $S(\mathbb{X} \times \mathbb{M})$ by $\mathcal{G}(S(\mathbb{X} \times \mathbb{M}))$. We define a partial ordering $\leqslant \mathcal{G}$ on $\mathcal{G}(S(\mathbb{X} \times \mathbb{M})), G_{1} \leqslant \mathcal{G} G_{2}$ if $G_{1}$ is subgraph of $G_{2}$ for $G_{1}, G_{2} \in \mathcal{G}(S(\mathbb{X} \times \mathbb{M}))$. A function $f: \mathcal{G}(S(\mathbb{X} \times \mathbb{M})) \rightarrow \mathbb{R}$ is said to be increasing if $f\left(G_{1}\right) \leqslant f\left(G_{2}\right)$ whenever $G_{1} \leqslant \mathcal{G} G_{2}$.

Fix $\lambda>0$ and let $\mathcal{P}$ be a homogeneous Poisson point process on $\mathbb{R}^{2}$ of intensity $\lambda$. The associated marked Poisson point process is denoted by $\eta$ having intensity measure $\lambda \ell_{2} \times Q$, where $Q:=\ell^{\mathbb{N} \times \mathbb{N}}$ and $\ell, \ell_{2}$ are Lebesgue measures on $[0,1], \mathbb{R}^{2}$ respectively. As described above we construct the random graphs $G(\eta, g), G_{x}(\eta \cup$ $\left.\left\{\left(x, T_{x}\right)\right\}, g\right)$ on the marked point process $\eta$, where $\left(x, T_{x}\right) \in \mathbb{R}^{2} \times \mathbb{M}$. For simplicity of notation we write $G, G^{x}$ and $G_{x}$ for the graphs $G(\eta, g), G\left(\eta \cup\left\{\left(x, T_{x}\right)\right\}, g\right)$ and $G_{x}\left(\eta \cup\left\{\left(x, T_{x}\right)\right\}, g\right)$ respectively. Clearly $G_{x}$ is a subgraph of $G^{x}$ and the two graphs $G$ and $G_{x}$ have the same distribution. The space of graphs is denoted by $\mathcal{G}\left(S\left(\mathbb{R}^{2} \times\right.\right.$ $\mathrm{M})$ ).

Let us now state and prove the FKG inequality in our setting. Let $f: \mathcal{G}\left(S\left(\mathbb{R}^{2} \times\right.\right.$ $\mathbb{M})) \rightarrow \mathbb{R}$ be a function. Recall the assumption $\int g(|x|) d x<\infty$ on the connection function which ensures that the graph has finite vertex degrees almost surely. We use the notation $f(\eta)$ to denote $f(G(\eta, g))$ and $E[f(\eta)]$ to denote its expected value. We say that a function $f \in L^{2}(\eta)$ if $E\left[f(\eta)^{2}\right]<\infty$. We denote the conditional expectation of $f$ given the vertex set $\mathcal{P}$ by $\tilde{E}[f(\eta)]:=E[f(\eta) \mid \mathcal{P}]$ where the averaging is over the distribution of the marks. A subset of $\mathbb{R}^{2}$ of the form $[a, b] \times[c, d]$ is called a box, where $a<b$ and $c<d, a, b, c, d \in \mathbb{R}$.

Theorem A.1 Suppose $B$ is box in $\mathbb{R}^{2}$. Let $f, h \in L^{2}(\eta)$ be two non-negative increasing functions that depend only on the edges intersecting the box $B$. Then the following FKG inequality holds,

$$
E[f(\eta) \cdot h(\eta)] \geqslant E[f(\eta)] E[h(\eta)] .
$$

Proof of Theorem A.l Consider a box $K$ such that $B \subset K$. We denote the restriction of the Poisson point process $\mathcal{P}$ to the set $K$ as $\mathcal{P}_{K}$ and the restriction of the marked process as $\eta_{K}$. Let $A_{K}$ be the event that there is no edge incident on $K^{c}$ that intersects $B$. Conditioning on the event $A_{K}$ and using the non-negativity of the functions leads to

$$
\begin{aligned}
E[f(\eta) \cdot h(\eta)] & \geqslant E\left[f(\eta) \cdot h(\eta) \mid A_{K}\right] P\left(A_{K}\right) \\
& =E\left[f\left(\eta_{K}\right) \cdot h\left(\eta_{K}\right)\right] P\left(A_{K}\right) \\
& =E\left[\tilde{E}\left[f\left(\eta_{K}\right) \cdot h\left(\eta_{K}\right)\right]\right] P\left(A_{K}\right) .
\end{aligned}
$$

The second step in (A.2) follows from the fact that conditioning on the event $A_{K}$ is equivalent to restricting the point process to the set $K$. An application of the discrete 
FKG inequality yields

$$
\tilde{E}\left[f\left(\eta_{K}\right) \cdot h\left(\eta_{K}\right)\right] \geqslant \tilde{E}\left[f\left(\eta_{K}\right)\right] \cdot \tilde{E}\left[h\left(\eta_{K}\right)\right] .
$$

Using (A.3) in (A.2) we obtain

$$
E[f(\eta) \cdot h(\eta)] \geqslant E\left[\tilde{E}\left[f\left(\eta_{K}\right)\right] \cdot \tilde{E}\left[h\left(\eta_{K}\right)\right]\right] P\left(A_{K}\right) .
$$

In the final step we shall apply the following FKG inequality for increasing functionals on Poisson point processes. Let $\mathcal{P}$ be a Poisson point process on a measurable space $(\mathcal{X}, \mathcal{F})$ with a $\sigma$-finite intensity measure $\mu$. A function $F: S(\mathbb{X}) \rightarrow \mathbb{R}$ is said to be increasing if $F(\mathcal{X} \cup\{x\}) \geqslant F(\mathcal{X})$ for any $\mathcal{X} \in S(\mathbb{X})$ and $x \in \mathbb{X}$. We say that $F \in L^{2}(\mathcal{P})$ if $E\left[F(\mathcal{P})^{2}\right]<\infty$.

Theorem A.2 (Theorem 20.4, Last and Penrose [36]) Let $F, H \in L^{2}(\mathcal{P})$ be two increasing functions. Then

$$
E[F(\mathcal{P}) \cdot H(\mathcal{P})] \geqslant E[F(\mathcal{P})] \cdot E[H(\mathcal{P})] .
$$

To apply the Theorem A.2 we need to show that $\tilde{E}\left[f\left(\eta_{K}\right)\right] \in L^{2}(\mathcal{P})$ and is increasing. Let $F\left(\mathcal{P}_{K}\right):=\tilde{E}\left[f\left(\eta_{K}\right)\right]$. That $F\left(\mathcal{P}_{K}\right) \in L^{2}(\mathcal{P})$ follows from the conditional version of the Jensen's inequality.

Recall that we write $f\left(\left(\eta_{K}\right)_{x}\right)$ for $f\left(G_{x}\left(\eta_{K}, g\right)\right)$ etc., and that $G_{x} \stackrel{d}{=} G$ we have for any $x \in K$

$$
\begin{aligned}
F\left(\mathcal{P}_{K}+\delta_{x}\right)-F\left(\mathcal{P}_{K}\right) & =\tilde{E}\left[f\left(\left(\eta_{K}\right)^{x}\right)\right]-\tilde{E}\left[f\left(\left(\eta_{K}\right)_{x}\right)\right] \\
& =\tilde{E}\left[f\left(\left(\eta_{K}\right)^{x}\right)-f\left(\left(\eta_{K}\right)_{x}\right)\right] \geqslant 0,
\end{aligned}
$$

where the last inequality follows from the fact that $f$ is increasing, i.e., $f\left(\left(\eta_{K}\right)^{x}\right) \geqslant$ $f\left(\left(\eta_{K}\right)_{x}\right)$.

Continuing the proof, by an application of Theorem A.2 to right hand side in (A.4) yields,

$$
E[f(\eta) \cdot h(\eta)] \geqslant E\left[f\left(\eta_{K}\right)\right] E\left[h\left(\eta_{K}\right)\right] P\left(A_{K}\right) .
$$

Since the connection function is integrable i.e., $\int g(|x|) d x<\infty$, every vertex in $B$ has finite degree almost surely. Hence, $P\left(A_{K}\right) \uparrow 1$ as $K \uparrow \mathbb{R}^{2}$. The result now follows by using this observation and the monotone convergence theorem in (A.7).

Acknowledgements The authors are thankful to Mathew D. Penrose and Yogeshwaran D. for many useful discussions and references. The authors also convey their gratitude to two anonymous reviewers for a careful reading of the article and suggesting several improvements and references that have significantly improved the article. 


\section{References}

1. Ahlberg, D., Tassion, V., Teixeira, A.: Sharpness of the phase transition for continuum percolation in $\mathbb{R}^{2}$. Probab. Th. Rel Fields 172(1), 525-581 (2018)

2. Aldous, D.J.: Scale-invariant random spatial networks. Electron. J. Probab. 19(15), 1-41 (2014)

3. Aldous, D.J., Shun, J.: Connected spatial networks over random points and a route-length statistic. Statist. Sci. 25(3), 275-288 (2010)

4. Aldous, D.J., Kendall, W.S.: Short-length routes in low-cost networks via Poisson line patterns. Adv. Appl Prob. 40(1), 1-21 (2008)

5. Alexander, K.S.: The RSW theorem for continuum percolation and the CLT for Euclidean minimal spanning trees. Ann. Appl. Prob. 6(2), 466-494 (1996)

6. Balberg, I., Binenbaum, N.: Computer study of the percolation threshold in a two-dimensional anisotropic system of conducting sticks. Phys. Rev B 28(7), 3799-3812 (1983)

7. Bollobás, B.: Random graphs, volume 73 of Cambridge Studies in Advanced Mathematics. Cambridge University Press, Cambridge (2001)

8. Bringmann, K., Keusch, R., Lengler, J.: Geometric inhomogeneous random graphs. Theor. Comput. Sci. 760, 35-54 (2019)

9. Cox, J.T., Durrett, R.: Limit theorems for the spread of epidemics and forest fires. Stoch. Process. Appl. 30(2), 171-191 (1988)

10. Daniels, C.J.E.: On the phase transition in certain percolation models. PhD Thesis, University of Bath (2016)

11. Deijfen, M., Hofstad, R.V.D., Hooghiemstra, G.: Scale-free percolation. Ann. Inst. H. Poincaré, Probab. Statist. 49(3), 817-838 (2013)

12. Deprez, P., Hazra, R.S., Wüthrich, M.V.: Inhomogeneous long-range percolation for real-life network modeling. Risks 3(1), 1-23 (2015)

13. Deprez, P., Wüthrich, M.V.: Scale-free percolation in continuum space. Commun. Math. Stat. 7, 269308 (2019)

14. Duminil-Copin, H., Tassion, V.: A new proof of the sharpness of the phase transition for Bernoulli percolation and the ising model. Commun. Math. Phy. 343(2), 725-745 (2016)

15. Erdős, P., Rényi, A.: On random graphs. Publ. Math. Debrecen 6, 290-297 (1959)

16. Erdős, P., Rényi, A.: On the evolution of random graphs. Publ. Math. Inst. Hungarian Acad. Sci. 5, 17-61 (1960)

17. Faggionato, A., Mimun, H.A.: Connection probabilities in Poisson random graphs with uniformly bounded edges. ALEA, Lat. Am. J. Probab. Math Stat. 16, 463-486 (2019)

18. Fitzner, R., Hofstad, R.V.D.: Mean-field behavior for nearest-neighbor percolation in $d>10$. Electron. J. Probab. 22, 1-65 (2017)

19. Fortuin, C., Kasteleyn, P.W., Ginibre, J.: Correlation inequalities on some partially ordered sets. Commun. Math Phy. 22, 89-103 (1971)

20. Franceschetti, M., Meester, R.: Random Networks for Communication. Cambridge University Press, Cambridge (2007)

21. Gilbert, E.N.: Random graphs. Ann. Math. Statist. 30(4), 1141-1144 (1959)

22. Gilbert, E.N.: Random plane networks. J. Soc. Indust. Appl. Math. 9(4), 533-543 (1961)

23. Gracar, P., Heydenreich, M., Mönch, C., Mörters, P.: Transience versus recurrence for scale-free spatial networks. In: International Workshop on Algorithms and Models for the Web-Graph, pp. 96-110. Springer (2020)

24. Grimmett, G.: Percolation. Springer, Berlin (1999)

25. Haenggi, M.: Stochastic Geometry for Wireless Networks. Cambridge University Press, Cambridge (2012)

26. Hara, T., Slade, G.: Mean-field critical behaviour for percolation in high dimensions. Commun. Math Phys. 128, 333-391 (1990)

27. Hara, T., Slade, G.: Mean-field behaviour and the lace expansion. In: Grimmett, G. (ed.) Probability and Phase Transition, NATO ASI Series (Series C: Mathematical and Physical Sciences), p. 420. Springer, Dordrecht (1994)

28. Heydenreich, M., Hofstad, R.V.D.: Progress in high-dimensional percolation and random graphs. Springer, Berlin (2017)

29. Heydenreich, M., Hofstad, R.V.D., Last, G., Matzke, K.: Lace expansion and mean-field behavior for the randomconnection model. arXiv:1908.11356v2 (2019) 
30. Hofstad, R.V.D.: Random graphs and complex networks. In: Cambridge Series in Statistical and Probabilistic Mathematics, vol. 1. Cambridge University Press, Cambridge (2017)

31. Hu, L., Hecht, G., Grüner, D.S.: Percolation in transparent and conducting carbon nanotube networks. Nano Lett. 4(12), 2513-2517 (2004)

32. Janson, S., Łuczak, T., Rucinski, A.: Random graphs. In: Wiley-Interscience Series in Discrete Mathematics and Optimization 2000. Wiley (2000)

33. Kesten, H.: The critical probability of bond percolation on the square lattice equals $1 / 2$. Comm. Math. Phys. 74(1), 41-59 (1980)

34. Kesten, H.: Percolation theory for mathematicians. Birkhäuser (1982)

35. Komjàthy, J., Lodewijks, B.: Explosion in weighted hyperbolic random graphs and geometric inhomogeneous random graphs. Stoch. Process. Appl. 130(3), 1309-1367 (2020)

36. Last, G., Penrose, M.D.: Lectures on the Poisson Process. Cambridge University Press, Cambridge (2017)

37. Liggett, T.M., Schonmann, R.H., Stacey, A.M.: Domination by product measures. Ann. Prob. 25(1), 71-95 (1997)

38. Meester, R.: Equality of critical densities in continuum percolation. J. Appl. Prob. 32(1), 90-104 (1995)

39. Meester, R., Roy, R.: Continuum Percolation. Cambridge University Press, Cambridge (1996)

40. Oskouyi, A., Sundararaj, U., Mertiny, P.: Effect of temperature on electrical resistivity of carbon nanotubes and graphene nanoplatelets nanocomposites. J. Nanotechnol. Eng. Med. 5(4), 044501044501 (2014)

41. Penrose, M.D.: Inhomogeneous random graphs, isolated vertices, and Poisson approximation. J. Appl. Prob., 55(1), 112-136 (2018)

42. Penrose, M.D.: Random Geometric Graphs. Oxford University Press, New York (2003)

43. Penrose, M.D.: On a continuum percolation model. Adv. Appl. Prob. 23(3), 536-556 (1991)

44. Pike, G.E., Seager, C.H.: Percolation and conductivity: A computer study. I. Phys. Rev. B 10(4), 1421-1434 (1974)

45. Roy, R.: The Russo-Seymour-Welsh theorem and the equality of critical densities and the "dual" critical densities for continuum percolation on $\mathbb{R}^{2}$. Ann. Prob. 18(4), 1563-1575 (1990)

46. Roy, R.: Percolation of poisson sticks on the plane. Probab. Th. Rel. Fields 89(4), 503-517 (1991)

47. Russo, L.: A note on percolation. Z.Wahrsch. verw Geb. 21, 39-48 (1978)

48. Schneider, R., Weil, W.: Stochastic and Integral Geometry. Springer, Berlin (2008)

49. Serre, P., Ternon, C., Stambouli, V., Periwal, P., Barona, T.: Fabrication of silicon nanowire networks for biological sensing. Sens. Actuators B 182, 390-395 (2013)

50. Seymour, P.D., Welsh, D.J.A.: Percolation probabilities on the square lattice. Ann. Discr Math. 3, 227-245 (1978)

51. Tassion, V.: Crossing probabilities for Voronoi percolation. Ann. Prob 44(5), 3385-3398 (2016)

52. Teixeira, A., Ungaretti, D.: Ellipses percolation. J. Stat. Phy. 168(2), 369-393 (2017)

Publisher's Note Springer Nature remains neutral with regard to jurisdictional claims in published maps and institutional affiliations.

\section{Affiliations}

\section{Srikanth K. Iyer ${ }^{1}$. Sanjoy Kr. Jhawar ${ }^{1}$}

Sanjoy Kr. Jhawar

sanjayjhawar@iisc.ac.in

1 Department of Mathematics, Indian Institute of Science, Bangalore, India 